\title{
Impact of injection rate ramp-up on nucleation and arrest of dynamic fault slip
}

\author{
F. Ciardo $^{a, b}$, A. P. Rinaldi ${ }^{a}$ \\ ${ }^{a}$ Swiss Seismological Service (SED) at ETH Zürich, Switzerland \\ ${ }^{b}$ Corresponding author: federico.ciardo@sed.ethz.ch
}

\begin{abstract}
Fluid injection into underground formations reactivates preexisting geological discontinuities such as faults or fractures. In this work, we investigate the impact of injection rate ramp-up present in many standard injection protocols on the nucleation and potential arrest of dynamic slip along a planar pressurized fault. We assume a linear increasing function of injection rate with time, up to a given time $t_{c}$ after which a maximum value $Q_{m}$ is achieved. Under the assumption of negligible shear-induced dilatancy and impermeable host medium, we solve numerically the coupled hydro-mechanical model and explore the different slip regimes identified via scaling analysis. We show that in the limit when fluid diffusion time scale $t_{w}$ is much larger than the ramp-up time scale $t_{c}$, slip on an ultimately stable fault is essentially driven by pressurization at constant rate. Vice versa, in the limit when $t_{c} / t_{w} \gg 1$, the pressurization rate, quantified by the dimensionless ratio $\frac{Q_{m} t_{w}}{t_{c} Q^{*}}$, does impact both nucleation time and arrest distance of dynamic slip. Indeed, for a given initial fault loading condition and frictional weakening property, lower pressurization rates delay the nucleation of a finite-sized dynamic event and increase the corresponding run-out distance approximately proportional to $\propto\left(\frac{Q_{m} t_{w}}{t_{c} Q^{*}}\right)^{-0.472}$. On critically stressed faults, instead, the ramp-up of injection rate activates quasi-static slip which quickly turn into a run-away dynamic rupture. Its nucleation time decreases non-linearly with increasing value of $\frac{Q_{m} t_{w}}{t_{c} Q^{*}}$ and it may precede (or not) the one associated with fault pressurization at constant rate only.
\end{abstract}

Keywords: Fault slip, Nucleation, Dynamic rupture, Induced seismicity 


\section{Introduction}

Anthropogenic fluid injection into underground formations is a common operation in many industrial applications. In the context of deep geothermal energy extraction, for instance, fluid is injected into targeted deep fault/fracture zones in order to enhance reservoir permeability and hence fluid circulation between injection and production wells (Giardini, 2009; Deichmann and Giardini, 2009). Among other applications that involve injection of fluid into subsurface there are hydraulic fracturing for oil and gas extraction from hydrocarbon reservoirs and wastewater disposal using deep wells (Warpinski and Teufel, 1987; Horton, 2012).

Although these engineering techniques are widely used, the injection of fluids into underground formations alters the local equilibrium of the Earth's crust, inducing micro-seismicity and, in some cases, large earthquakes (Horton, 2012; Ellsworth, 2013; Kim, 2013; Keranen et al., 2014; Weingarten et al., 2015). Significant earthquakes have been directly correlated with the injection activities, among these the Pohang earthquake of 2017 in South Korea with $M_{w}=5.5$ (Kim et al., 2018; Grigoli et al., 2018; Yeo et al., 2020), the four $M_{w}=3$ events in Basel, Switzerland, between 2006 and 2007 (Deichmann and Giardini, 2009; Goertz-Allmann et al., 2011), an event of $M_{w}=3.5$ in the city of St. Gallen, Switzerland, back in 2013 (Diehl et al., 2014; Edwards et al., 2015; Diehl et al., 2017) and the $M_{w}=5.7$ earthquake near Prague, Oklahoma, in 2011 (Keranen et al., 2013; Sumy et al., 2014). Many numerical and theoretical models have been developed in order to investigate the impact of operational design parameters, such as injection pressure or injection rate, on fault slip activation and earthquakes nucleation upon fluid injection. Most of them are based on a Rate- and Statedependent friction model and, therefore, are well suited to explain features of earthquake cycles and seismicity rates. For example, Dempsey and Riffault (2019) used a pressure diffusion model coupled to R\&S friction model to show that a reduction in injection rate may lead to a decrease in the seismicity rate in Oklahoma (USA). A similar result has been obtained by Lagenbruch and Zoback (2016) using instead a statistical model calibrated over many injection induced-earthquakes in Oklahoma. Using a poroelastic model incorporating R\&S friction, Barbour et al. (2017) observed that in Oklahoma a variable injection rate may lead to a larger seismicity rate in- 
creases compared to the one under constant injection rate (for an equivalent injected volume). Chang et al. (2018), instead, studied the effect of injection rate variation on seismicity rate post shut-in and they showed that a gradual reduction of injection rate minimises the post-injection seismicity rate. Using a Dietrich-Ruina heterogeneous 2D fault, Almakari et al. (2019) investigated the effect of injection scenario not only in terms of seismicity rate, but also in terms of magnitude content. They showed that the total seismic moment increases with both maximum pressure and pressure rate and that the total number of induced seismic events is controlled by the maximum pressure. A recent study of Rudnicki and Zhan (2020) on a spring-block model shows that larger pressurization rates stabilize fault slip events due to rate and state friction.

The role of injection design parameters on fault slip behaviour has been extensively investigated also in many laboratory experiments. Among others, Wang et al. (2020) showed that fault slip propagation is manly governed by fluid pressurization rate rather than injection pressure. French et al. (2016), instead, observed that fluid pressurization is less effective than mechanical changes in the fault normal stress at initiating accelerated slip events. The effect of fluid pressure oscillations on fault slip stability has been investigated by Noël et al. (2019) via a triaxial laboratory experiment. They showed that perturbations caused by pore fluid oscillations promote seismic slip and that seismic activity along a fault increases for increasing oscillation's amplitudes. Despite the numerous studies on the effects of injection parameters on earthquakes nucleation and occurrence along faults, the impact of pressurization rate on the onset of dynamic fault slip remains still elusive. Garagash and Germanovich (2012) investigated extensively the conditions of nucleation and arrest of dynamic fault slip on a frictional weakening pressurized fault. Their generic findings, however, are valid only for two types of fault pressurizations, constant over-pressure and constant injection rate, which are oversimplification of many injection protocols commonly used in industrial applications. In numerous fault reactivation experiments, for instance related to deep geothermal energy exploitation, the injection protocol consists of one (or more) stimulation cycle, where the controlled injection rate or injection pressure increases in time (typically with stair-like increments), up to reach a steady state regime, followed then by a shut-in phase (Hofmann et al., 2018). In the hydraulic stimulation experiments conducted in Grimsel Test Site, Switzerland, back in 2017 for example, the injection protocol consisted of 4 injection cycles in which either injection pressure or injection flow rate 
was increased in a stepwise manner, before reaching a plateau and shut-in phase (Villiger et al., 2020). A similar trend of injection rate was also used during the hydraulic stimulation of Enhanced Geothermal System in the city of Basel, Switzerland (2006) (Häring et al., 2008) and in the more recent EGS project in Pohang, South Korea (Hofmann et al., 2018), to cite a few examples.

In this contribution, we extend the model of Garagash and Germanovich (2012) to account for an initial ramp-up of injection rate in time and investigate its effect on dynamic fault slip nucleation and arrest. We approximate the step-wise increase of injection rate adopted in standard injection protocols using a simple linear increasing function with time, followed by a maximum plateau (the shut-in phase is out of context and thus is not considered in this work). This choice represents a good approximation when the time scale of each increment is much larger that the corresponding one of each step (and thus a linear increasing function is a reasonable approximation). We solve the two-dimensional hydro-mechanical problem numerically and verify the results with theoretical predictions. The goal of this study is to evaluate the effects of the pressurization rate, quantified by the injection rate ramp-up variation, on the nucleation and arrest of a seismic rupture on a frictional weakening planar fault.

\section{Fault model}

We consider a planar fault embedded in an isotropic, homogeneous and unbounded elastic medium under plane-strain conditions (see Figure 1). The fault is subjected to an ambient pore-pressure $p_{o}$ and a uniform far-field stress state that resolved on the fault plane result in an effective normal $\sigma_{o}^{\prime}=\sigma_{n}-p_{o}$ and shear $\tau^{o}$ stress component. Such a uniform ambient stress state, typical of a limited fault extent compared to the background in-situ gradient, is perturbed via a point source injection of volumetric flow rate $Q(t)\left[L^{2} / T\right]$ directly in the middle of the fault (specifically at $x=0$ ). In order to investigate the effect of injection rate ramp-up on fault slip stability, we consider a linear increase of injection flow rate in time, followed by a plateau after a given time $t_{c}$ (the shut-in phase is out of scope here, hence it will not be considered - see Figure 1). This parametrisation represents an approximation of many standard fault injection protocols used in hydroshearing stimulation of fractured reservoirs, in which the design (constant) value of injection rate is reached upon stair-like increments. 


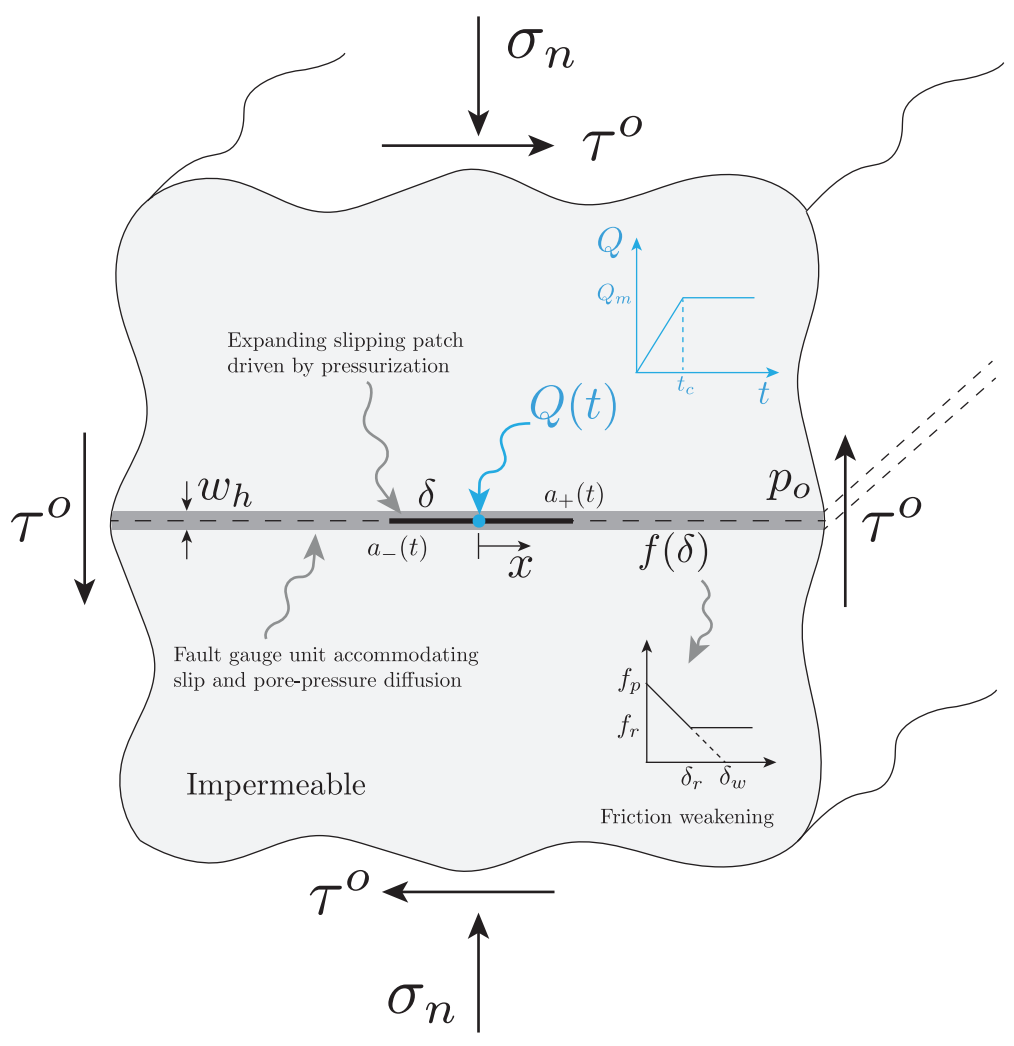

Figure 1: Plane-strain fault model subjected to a far-field stress state and fluid injection. The conductive planar fault is embedded into an homogeneous, isotropic and linear-elastic medium characterized by a negligible hydraulic diffusivity. The thin fault gouge unit, therefore, accommodates accelerating slip due to friction weakening condition and porepressure diffusion.

Prior fluid injection, we assume that the fault is in static equilibrium with the uniform in-situ stress state (locked status). This tacitly assumes that there is no effect of remote plates loading that would cause steady movements with slow energy release (creep) (Chen and Bürgmann, 2017). The ambient equilibrium is violated throughout pressurization, during which pore-pressure perturbation diffuses along the fault plane and activates a symmetric shear crack of length $2 a$. This scenario of mechanically weak, thin fault gouge unit accommodating slip and pore fluid flow may be representative of an immature deep fault, whose hydraulic conductivity is much larger than the relatively undamaged rock around it (and thus impermeable host medium is 
a reasonable assumption).

Before presenting the governing equations, it must be noted that we assume normal stresses positive in compression and shear stresses positive for clockwise rotation. Furthermore, thermal effects, dilatancy/compaction during shear deformations and poroelastic stress changes in the surrounding medium are neglected.

2.1. Governing equations for quasi-static slip development driven by porepressure diffusion

\subsubsection{Static equilibrium and constitutive law for frictional slip}

We consider the activation and propagation of a symmetric shear crack of length $2 a$ driven by pore fluid flow inside the conductive fault plane. The shear stress $\tau$ at a given time $t$ and position $x$ on the slip surface is linearly related to slip $\delta$ (or shear displacement discontinuity) within the slipping region via the following quasi-static elastic equilibrium equation

$$
\tau(x, t)=\tau^{o}-\frac{E_{p}}{4 \pi} \int_{a_{-}(t)}^{a_{+}(t)} \frac{\partial \delta(\zeta, t) / \partial \zeta}{\zeta-x} \mathrm{~d} \zeta,
$$

where $a_{+}$and $a_{-}$are respectively the positive and negative positions of the crack tips, $\tau^{o}$ is the uniform background shear stress, $E_{p}=\frac{2 G}{1-\nu}$ is the planestrain Young's modulus (with $G$ and $\nu$ being shear modulus and Poisson's ratio, respectively), $\partial \delta(\zeta, t) / \partial \zeta$ is the shear dislocation density along the crack that must satisfy the following condition

$$
\lim _{\zeta \rightarrow a_{ \pm}(t)} \frac{\partial \delta(\zeta, t)}{\partial \zeta} \sqrt{a \mp \zeta}=0
$$

in order to remove stress singularity at crack tips (Uenishi and Rice, 2003), and $\frac{1}{\zeta-x}$ is the non-local elastic kernel. For merely planar frictional problems that do not account for dilatancy or compaction during crack propagation, the elastic kernel affects only the shear stress distribution along the fault plane, while the uniform total normal stress $\sigma_{n}$ remains constant.

Inside the sliding region, the force balance requires that the shear stresses must equal the available frictional resistance which we assume here to obey the Mohr-Coulomb yielding criterion (without cohesion), accounting for a slip weakening of friction coefficient

$$
\tau(x, t)=f(\delta)\left(\sigma_{n}-p(x, t)\right), \quad|x| \leq a(t)
$$


where $\left(\sigma_{n}-p(x, t)\right)=\sigma_{n}^{\prime}(x, t)$ is the local effective normal stress, strictly function of pore-pressure evolution $p(x, t)$ inside the fault, and $f(\delta)$ is the slip weakening friction coefficient

$$
f(\delta)= \begin{cases}f_{p}-\frac{\left(f_{p}-f_{r}\right)}{\delta_{r}} \cdot \delta & \delta \leq \delta_{r} \\ f_{r} & \delta>\delta_{r}\end{cases}
$$

The frictional resistance, therefore, weakens linearly with shear deformations $\delta$, from a peak value associated with peak friction coefficient $f_{p}$, to a residual value, after sufficiently large slip $\delta_{r}$ (such that the friction coefficient drops to its residual value $f_{r}$ ). Although more complicated frictional laws can be considered in (3), we use here the simple piece-wise linear weakening of friction coefficient with slip, which can be shown to be a good approximation of the phenomenological rate- and state- friction law at large slip rates and for small values of $a / b$ (Uenishi and Rice, 2003; Rubin and Ampuero, 2005), or when $\Delta f_{p} / b \gg 1$, with $\Delta f_{p}$ being the peak change of friction coefficient from the steady-state value (Garagash, 2021).

\subsubsection{Pore-pressure diffusion}

Under the assumption of negligible fluid leak-off in the surrounding elastic medium, fluid flow is confined within the fault gouge unit characterised by a constant hydraulic aperture $w_{h}$. Upon injection of volumetric flow rate $Q(t)$ at $x=0$, the diffusion of pore fluid over-pressure $\bar{p}(x, t)=p(x, t)-p_{o}$ is governed by the width-averaged fluid mass conservation equation

$$
w_{h} c_{f} \frac{\partial \bar{p}}{\partial t}+\frac{\partial w_{h} v}{\partial x}=0,
$$

where $c_{f}\left[M^{-1} T L^{2}\right]$ is a parameter that combines pore fluid compressibility and pore space expansivity and $v$ is the gap-averaged fluid flow velocity given by the Poiseuille's law

$$
v=-\frac{k_{f}}{\mu} \frac{\partial \bar{p}}{\partial x},
$$

where $k_{f}\left[L^{2}\right]$ is the longitudinal fault permeability and $\mu=12 \mu^{\prime}\left[M T^{-1} L^{-1}\right]$ is a viscosity parameter (with $\mu^{\prime}$ as dynamic viscosity of the fluid). The point injection boundary condition requires that

$$
w_{h} v= \pm \frac{Q(t)}{2}, \quad \text { at } \quad x=0^{ \pm}
$$


where the volumetric flow rate $Q(t)\left[L^{2} T^{-1}\right]$ increases linearly with time $t$, up to reach a plateau after a given time $t_{c}$, i.e.

$$
Q(t)= \begin{cases}\frac{Q_{m}}{t_{c}} \cdot t & t \leq t_{c} \\ Q_{m} & t>t_{c}\end{cases}
$$

Since we neglect dilatation/compaction of fault gouge unit during shear crack propagation, fault permeability $k_{f}$ and hydraulic aperture $w_{h}$ remain constant throughout pressurization. Equations (5) and (6), therefore, reduce to the well-known parabolic diffusion equation

$$
\frac{\partial \bar{p}}{\partial t}-\alpha \frac{\partial^{2} \bar{p}}{\partial x^{2}}=0
$$

that govern over-pressure diffusion inside the fault conduit characterized by a constant hydraulic diffusivity $\alpha=\frac{k_{f}}{c_{f} \cdot \mu}\left[L^{2} T^{-1}\right]$. Using specific boundary and initial conditions representative of the particular injection scenario and its time history, equation (9) can be solved analytically for the spatial and temporal evolution of pore-fluid over-pressure along the fault plane $\bar{p}(x, t)$ (see Appendix A for full details). During the ramp-up phase of injection rate, i.e. for $t \leq t_{c}$, the over-pressure evolution in function of time $t$ and the normalized coordinate $\xi=\frac{x}{\sqrt{4 \alpha t}}$ is given by

$\bar{p}\left(\xi, t \leq t_{c}\right)=\underbrace{\left(\frac{2 Q_{m} \mu \sqrt{\alpha} t^{3 / 2}}{3 k_{f} \sqrt{\pi} t_{c} w_{h}}\right)}_{\Delta P(t)} \cdot \underbrace{\left(\mathrm{e}^{-\xi^{2}}\left(1+\xi^{2}\right)-\sqrt{\pi}|\xi|\left(\frac{3}{2}+\xi^{2}\right) \operatorname{Erfc}(|\xi|)\right)}_{\Psi(\xi)}$

where Erfe is the complementary error function. Notice that the analytical solution (10) is expressed as a product two independent functions, $\Delta P(t)$ and $\Psi(\xi)$, which identify respectively the maximum over-pressure evolution at injection point and its instantaneous spatial distribution.

The pore-fluid evolution after the ramp-up phase, instead, is governed by the following equation (Cole et al., 2011)

$\bar{p}\left(x, t>t_{c}\right)=\int_{-\infty}^{\infty} G\left(x-x^{\prime}, t-t_{c}\right) \cdot \bar{p}\left(\frac{x^{\prime}}{\sqrt{4 \alpha t_{c}}}, t_{c}\right) \mathrm{d} x^{\prime}+\frac{\alpha Q_{m} \mu}{w_{k} k_{f}} \int_{t^{\prime}=t_{c}}^{t} G\left(x, t-t^{\prime}\right) \mathrm{d} t^{\prime}$ 
where $\bar{p}\left(\frac{x^{\prime}}{\sqrt{4 \alpha t_{c}}}, t_{c}\right)$ denotes the over-pressure distribution at time $t_{c}$ and $G\left(x-x^{\prime}, t-t^{\prime}\right)$ is the fundamental heat conduction solution valid for an infinite one-dimensional body subjected to an instantaneous point source (also called Green's function) (Carslaw and Jaeger, 1959). We revert the reader to Appendix A for its analytical expression. It is worth mentioning that the solution (11) is valid for $t>t_{c}$ and it takes into account the whole injection history during the ramp-up phase. If $t_{c}$ vanishes, then we recover the analytical solution of pressurization at constant injection rate (see Appendix A).

In this contribution, we assume that maximum over-pressure occurring at injection point $x=0$ remains always below the ambient effective normal stress $\sigma_{o}^{\prime}=\sigma_{n}-p_{o}$ applied on the fault plane, i.e.

$$
\frac{\bar{p}(0, t)}{\sigma_{o}^{\prime}}<1, \quad \forall t
$$

implying that the minimum principal effective stresses $\sigma_{n}^{\prime}(x, t)$ remain always compressive (positive) throughout pressurization and hydraulic fracturing type of failure never occurs (which would require the full coupling between flow and elastic deformations).

The along-fault pore-pressure diffusion changes the local effective normal stresses and hence drives the symmetric slip propagation when the MohrCoulomb criterion (3) is locally violated. Shear deformations, instead, do not affect pore-pressure evolution since shear-induced dilatancy or compaction is neglected and so fault permeability / porosity changes too. Although this assumption is debatable, since such inelastic deformations do affect slip stability on a planar fault with frictional weakening properties (Garagash and Rudnicki, 2003; Zhang et al., 2005; Ciardo and Lecampion, 2019), we want to minimise the complexities in the model and focus solely on the effect of injection rate ramp-up on the potential nucleation and arrest of dynamic slip. The hydro-mechanical model, therefore, is only one-way coupled and it is equivalent to the one proposed by Garagash and Germanovich (2012), with the difference that the point injection volumetric rate is not constant in time but changes according to (8).

We have introduced in the model an additional parameter $t_{c}$, therefore we expect another dimensionless parameter governing the hydro-mechanical fault's response (on top of the ones introduced by Garagash and Germanovich (2012)). For sake of completeness we present and discuss in the next section 
all the dimensionless governing parameters resulting from scaling analysis (see Appendix B for more details), as well realistic values that are then used in the numerical simulations.

\subsection{Dimensionless governing parameters}

Upon normalization of all the governing equations (1-8) following Uenishi and Rice (2003); Garagash and Germanovich (2012) (see Appendix B), the hydro-mechanical fault response depends only on four dimensionless parameters:

- Stress criticality $\frac{\tau^{o}}{\tau_{p}}$, which represents the closeness of the ambient fault stress state to failure (and thus to the peak shear strength $\tau_{p}=f_{p} \sigma_{o}^{\prime}$ ). Levandowski et al. (2018) claims that stress criticality is the most important factor for induced earthquakes hazard. Favourably oriented frictional weakening faults with respect to the in-situ stress field, typically characterised by a large stress criticality $\left(\frac{\tau^{o}}{\tau_{p}} \lesssim 1\right)$, are very susceptible to host run-away seismic ruptures. Indeed, a little stress perturbation is sufficient to re-activate slip and its velocity propagation tends to diverge rapidly due to friction weakening and possibly other weakening mechanisms, such us flash-heating and thermal pressurization (Viesca and Garagash, 2015). Garagash and Germanovich (2012) have shown via a stability analysis that critically stressed pressurized faults, for which the relation $\tau^{o}>\tau_{r}=f_{r} \sigma_{o}^{\prime}$ is strictly satisfied, host always the nucleation of an unabated dynamic event. Such a run-away rupture, however, can be suppressed when shear-induced dilatancy kicks-off and dilatant hardening stabilises slip propagation (Lockner and Byerlee, 1994; Segall et al., 2008; Ciardo and Lecampion, 2019). Critically stressed faults in seismogenic zones have been observed in Oklahoma and Southern Kansas (Qin et al., 2019), in the German continental deep drillhole (KTB) (Ito and Zoback, 2000) and in central California along the San Andreas fault system (Zoback et al., 1987; Rice, 1992) to cite a few examples.

On the other hand, fault zones not favourably oriented to the local in-situ stress field are characterised by low stress criticality $\left(\frac{\tau^{o}}{\tau_{p}} \gtrsim 0\right)$, which implies that larger over-pressures are required to activate slip. By making the analogy with critically stressed pressurized faults exhibiting 
linear slip-weakening behaviour, low stress criticality and $\tau^{o}<\tau_{r}$ implies a quasi-static, stable slip propagation with eventually a nucleation and arrest of a dynamic event at large pressurization time (Garagash and Germanovich, 2012).

The role of the initial effective stress state on the nature of seismicity has been also investigated in fault lab experiments by Passelègue et al. (2020), where they show that faults with similar frictional properties can rupture at both slow and fast rupture velocity depending on their initial stress criticality, in agreement with theoretical predictions based on linear elastic fracture mechanics (LEFM).

- Friction weakening ratio $\frac{f_{r}}{f_{p}}=1-\frac{\delta_{r}}{\delta_{w}}$ that governs the shear stress drop within the crack tips (with $\delta_{w}=\frac{f_{p}}{\left(f_{p}-f_{r}\right)} \delta_{r}$ being the amount of slip at which the friction coefficient goes to zero if an unlimited linear slip-weakening friction law is considered). According to slip laboratory experiments of granite intact specimens, the slip weakening distance $\delta_{r}$ is approximately half a millimetre (Rice, 1980), but it can drop even below $0.1 \mathrm{~mm}$ (Wong, 1986). Similarly, $\delta_{w}$ is on the order of fault's asperities and thus ranges between 0.1 and $10 \mathrm{~mm}$. The friction weakening ratio, therefore, can assume any values within the interval $(0,1)$.

- Normalized maximum injection rate $\frac{Q_{m}}{Q^{*}}$, where $Q^{*}=\frac{2 \sigma_{o}^{\prime} w_{h} k_{f}}{a_{w} \mu}$ is the characteristic injection rate scale and $a_{w}=\frac{E_{p}}{2 \tau_{p}} \delta_{w}$ is the slipping patch length-scale. Its value is typically in the order of a meter, but roughly one order of magnitude of variation is plausible due to the variation of $\delta_{w}$ with fault's roughness and $\sigma_{o}^{\prime}$ with hydrogeological conditions. In normally pressurized formations, typically located in the Earth's upper crust and characterised by lithostatic gradient and hydro-static pore-pressure conditions (Brace and Kohlstedt, 1980; Grawinkel and Stockhert, 1997), $\sigma_{o}^{\prime}$ may be a fraction of megapascals, while a boost to hundred of megapascals may be obtained in over-pressurized formations located below the fluid retention depth (Suppe, 2014). Assuming a fluid viscosity parameter $\mu \sim 10^{-3} \mathrm{~Pa} \cdot \mathrm{s}$ (water), a fault gouge permeability $k_{f}$ in the order of $\sim 10^{-16} \mathrm{~m}^{2}$ (Wibberley et al., 2008) and 
hydraulic width $w_{h}$ of few millimetres, the injection rate characteristic scale $Q^{*}$ ranges between $\sim 10^{-9} \mathrm{~m}^{2} / \mathrm{s}$ and $10^{-6} \mathrm{~m}^{2} / \mathrm{s}$. If we assume that the out-of-plane fault length is $\sim 1 \mathrm{~km}$, then the volumetric injection rate scale would range between a fraction to few litres per second. The maximum injection rate $Q_{m}$ is a design parameter that can vary considerably from tens or hundreds litres per second in hydraulicfracturing operations (see (Holland, 2013) for one example where $>160$ l/s were injected in south-central Oklahoma), to few or fractions of litres per second for hydro-shearing stimulations (see for instance hydroshearing experiments in Grimsel Test Site, Switzerland, where the maximum injection rate never exceeded $0.5 \mathrm{l} / \mathrm{s}$ (Villiger et al., 2020)).

The dimensionless parameter $\frac{Q_{m}}{Q^{*}}$, therefore, can assume relatively low or large values depending on the specific problem configuration. Since hydraulic fracturing type of failure is not considered in our model, all the numerical results that will be presented later are characterised by a relatively low/moderate value of $\frac{Q_{m}}{Q^{*}}$.

- $\frac{t_{c}}{t_{w}} \rightarrow$ ratio between the ramp-up time scale and along-fault diffusion time-scale $t_{w}=\frac{a_{w}^{2}}{4 \alpha}$. This ratio may vary considerably due to the variation of slipping patch length-scale $a_{w}$ previously discussed, the specific fault hydraulic properties considered as well as the choice of the parameter $t_{c}$. Assuming a compressibility parameter $c_{f} \sim 0.5 \mathrm{GPa}^{-1}$ (Wibberley, 2002) and the hydraulic parameters previously defined (albeit the fault longitudinal permeability may range within the interval $10^{-19}-10^{-15} \mathrm{~m}^{2}$ ), we estimate an hydraulic diffusivity of $\alpha \sim 2 \cdot 10^{-4}$ $\mathrm{m}^{2} / \mathrm{s}$, which leads to a diffusion time scale of $t_{w} \sim 21$ min for $a_{w}=1$ $\mathrm{m}$.

The parameter $t_{c}$ may be in the order of several minutes for relatively fast fault pressurizations (see for instance one cycle of the injection protocol used for one hydro-shearing stimulation in Grimsel, Switzerland (Amann et al., 2018)), but it may get up to several hours or few days for relatively slow pressurizations (see for instance the injection protocol used for hydraulic stimulation of Pohang Enhanced Geothermal System, 2017 (Yeo et al., 2020; Hofmann et al., 2018)). The ratio $t_{c} / t_{w}$, therefore, may be very large, in the order 1 or possibly very small. 
In the following we explore the parameter space identified by these dimensionless ratios via numerical simulations. We vary them systematically in order to investigate their impact on the nucleation and arrest (if occur) of dynamic fault slip.

\section{Numerical results}

We use a fast boundary element based solver in order to solve numerically the one-way coupled hydro-mechanical problem (1-11) (see details in (Ciardo et al., 2020)). It is suited for 2D non-linear geo-mechanical problems involving localized inelastic deformations along pre-existing structural discontinuities, such as faults or fractures. The elasto-static balance of momentum (1) is discretized using displacement discontinuity method that, together with the discretized form of shear weakening Mohr-Coulomb criterion (3), lead to a non-linear system of equations for the unknowns inelastic deformations (or displacement discontinuities) and effective tractions. For a given porepressure history calculated using (10) and (11) (with the latter evaluated numerically using a trapezoidal quadrature rule), such a resulting system is solved iteratively using fixed point iterations combined with under-relaxation (Quarteroni et al., 2000), and adopting a fully implicit integration scheme in time. A speed up of computations is obtained via hierarchical approximation of the fully populated elasticity matrix (on top of memory reduction), together with the use of an adequate block pre-conditioner that improves spectral properties of the resulting matrix of coefficients (see full details in (Ciardo et al., 2020)).

All the numerical results that will be presented in the following are obtained by considering a sufficiently long planar fault such that $L_{o} / a_{w}=40$, with $L_{o}$ being its half-length. The planar fault is then uniformly discretized with $2 \cdot 10^{3}$ equal-sized straight elements, resulting in having 50 elements inside the slip weakening region near crack tips (sufficient to accurately capture the model non-linearity).

\subsection{Ultimately stable fault $\left(\tau^{o}<\tau_{r}\right)$}

Firstly, we present the case of fluid injection into an ultimately stable fault characterised by $\tau^{o} / \tau_{r}<1$, where $\tau_{r}=f_{r} \sigma_{o}^{\prime}$ is the residual frictional strength prior pressurization. This condition automatically implies that the fault is far from being critically stressed, and thus the ratio $\tau^{o} / \tau_{p}$ is kept relatively low. By fixing the frictional weakening ratio to $f_{r} / f_{p}=0.6$, we investigate the 


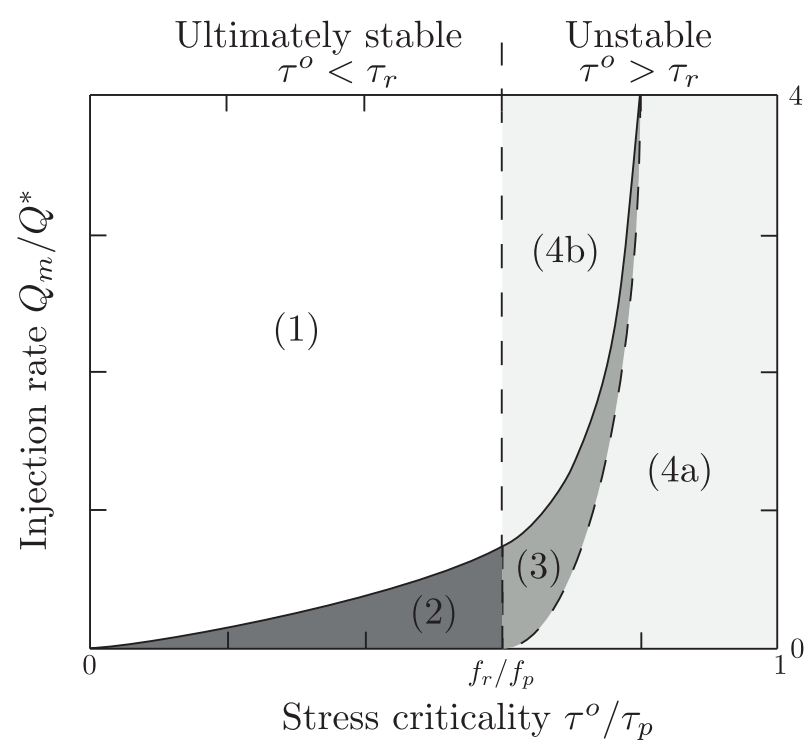

Figure 2: Map of slip regimes for a frictional weakening pressurized fault (adapted from Garagash and Germanovich (2012)), as function of stress criticality $\tau^{o} / \tau_{p}$, friction weakening ratio $f_{r} / f_{p}$ and normalized (constant) injection rate $Q_{m} / Q^{*}$. Region (1) corresponds to the scenario of an ultimately stable fault in which pressurization leads always to quasistatic (stable) slip propagation. Region (2) corresponds to the case of quasi-static slip propagation, followed by a nucleation and arrest of dynamic slip. Regions (4b) and (4a), instead, represent the scenarios of an unstable fault exhibiting quick quasi-static crack propagation followed by an unabated dynamic rupture, whose nucleation is affected or not by residual friction coefficient $f_{r}$, respectively. Finally, in region (3) the nucleation of a run-away rupture on an unstable fault is preceded by a finite-sized seismic event.

fault's hydro-mechanical response for different values of normalized maximum injection rate $Q_{m} / Q^{*}$, in the two plausible limiting scenarios of $t_{c} / t_{w} \ll 1$ and $t_{c} / t_{w} \gg 1$.

\subsubsection{Fast ramp-up: the limit when $t_{c} / t_{w} \ll 1$}

If the diffusion time-scale $t_{w}$ is much larger than the ramp-up time $t_{c}$ of injection rate, slip activation and propagation occurs when the injection rate has already reached its maximum value $Q_{m} / Q^{*}$. The quick ramp-up of injection rate has a negligible effect on pore-pressure evolution and the hydro-mechanical fault response is essentially driven by injection at constant volumetric rate. Garagash and Germanovich (2012) studied extensively the effect of such a type of fault pressurization on the nucleation and potential arrest of a dynamic event. They came up with a slip regimes diagram 

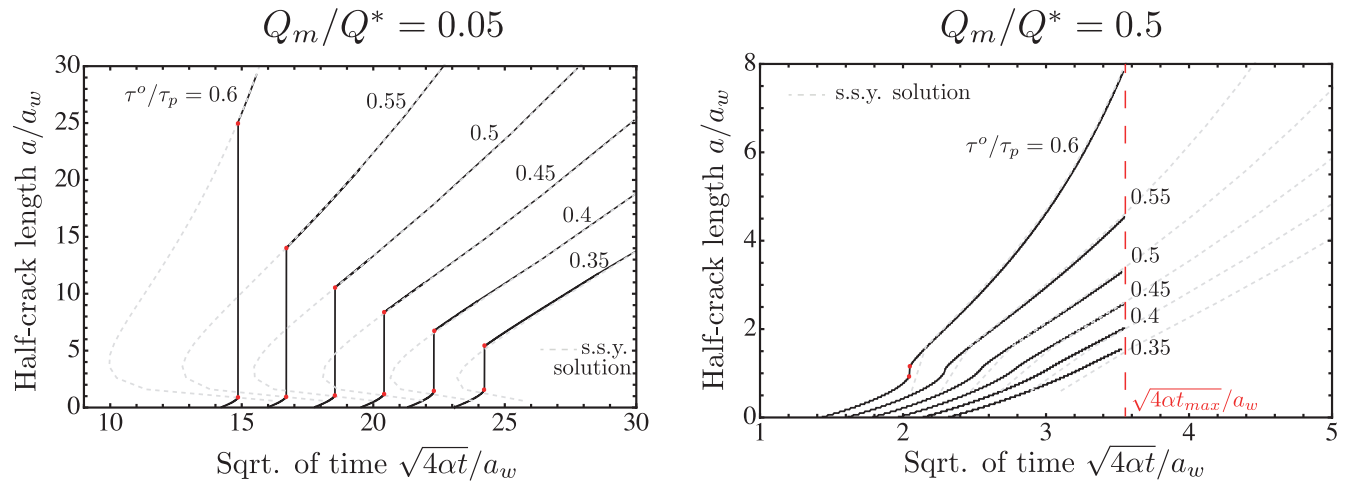

Figure 3: Time evolution of normalized half-crack length $a / a_{w}$ (black solid lines) as function of various stress criticality values $\tau^{o} / \tau_{p}$, for two values of maximum injection rates $Q_{m} / Q^{*}=0.05,0.5$ and $t_{c} / t_{w}=0.01 \ll 1$. The friction weakening ratio is set to $f_{r} / f_{p}=0.6$, resulting in ultimately stable conditions, i.e. $\tau^{o}<\tau_{r}$ for each value of ambient fault loading condition. Grey dashed lines represent the small scale yielding (s.s.y.) solutions (see Appendix C) associated with constant injection rate type of pressurization, which represents a good approximation of the hydro-mechanical fault response when $t_{c} / t_{w} \ll 1$. In this particular example, indeed, the normalized ramp-up time is $\sqrt{4 \alpha t_{c}} / a_{w}=0.1$ and thus shear crack activation and propagation is essentially driven at constant injection rate. Red dots, instead, denote the nucleation and arrest of dynamic slip.

reported in Figure 2, in which a frictional weakening planar fault may experience a finite-sized dynamic event (region (2)), a run-away rupture (affected or not by the residual friction coefficient $f_{r}$, region (4b) and (4a) respectively), a finite-sized dynamic event followed by an unabated seismic rupture (region (3)), or only aseismic slip (region (1)). This depends on the particular set of dimensionless parameters that identifies a specific initial fault loading condition, pressurization and frictional property. Their theoretical and semi-analytical results, therefore, provide benchmark solutions for our numerical results.

Under ultimately stable conditions (i.e. $\tau^{o}<\tau_{r}$, left side of Figure 2), Garagash and Germanovich (2012) proved via a stability analysis that a fault experiences a stable, quasi-static growth of slipping patch throughout sustained pressurization (i.e. aseismic slip). However, for sufficiently low injection rates $Q_{m} / Q^{*}$, the fault exhibits a finite-sized dynamic event, whose nucleation time $t_{n}$ is considerably larger than fluid diffusion time-scale $t_{w}$ (and thus $\left.t_{c} \ll t_{w} \ll t_{n}\right)$. These considerations are also confirmed with our numeri- 

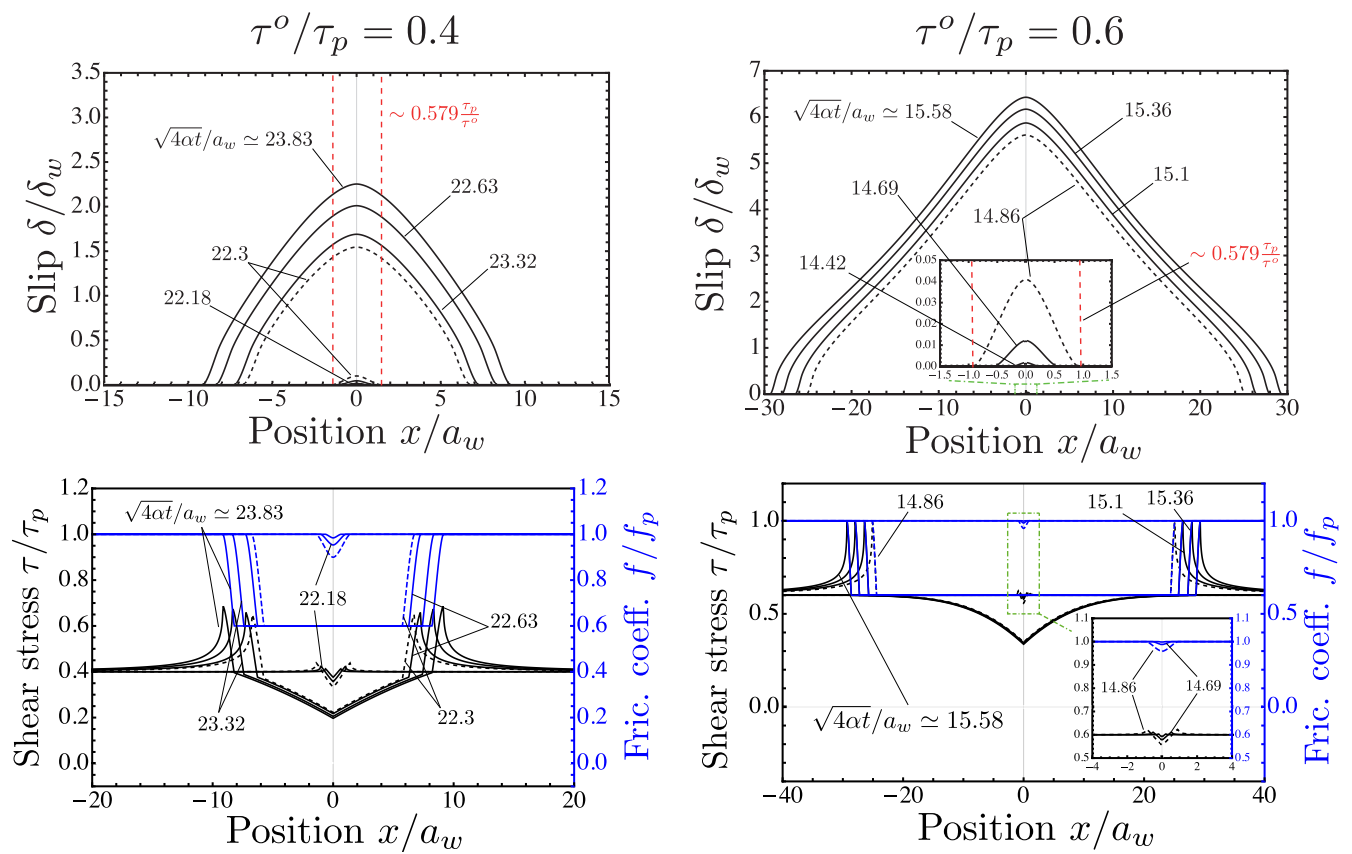

Figure 4: Normalized slip $\delta / \delta_{w}$, shear stress $\tau / \tau_{p}$ and friction coefficient $f / f_{p}$ profiles at different normalized time snapshots, for the case of maximum injection rate $\frac{Q_{m}}{Q^{*}}=0.05$, ramp-up time scale $t_{c} / t_{w}=0.01 \ll 1$ and two stress criticality values, i.e. $\tau^{o} / \tau_{p}=0.4$ and 0.6 (ultimately stable faults as $f_{r} / f_{p}=0.6$ ). Dashed blue and black lines correspond to the numerical solutions at nucleation time $t_{n}$ (or in normalized form $\frac{\sqrt{4 \alpha t_{n}}}{a_{w}}$ ), while dashed vertical red lines denote the asymptotic solution (13) provided by Garagash and Germanovich (2012).

cal results reported in Figure 3, where the normalized time evolution of half crack length $a / a_{w}$ is displayed for two different values of $Q_{m} / Q^{*}=0.05,0.5$ and different values of low/moderate stress criticality $\tau^{o} / \tau_{p}$ (with the ratio $t_{c} / t_{w}$ equal to 0.01 and $\tau^{o}$ always below or equal $\tau_{r}$ ).

For the case of low injection rate $Q_{m} / Q^{*}=0.05$, a nucleation of dynamic event followed by an arrest always occurs for each value of stress criticality considered, with larger dynamic run-out distances for increasing values of $\tau^{o} / \tau_{p}$ (see Figure 3-left). Since the ambient effective stress states applied on the fault plane are far from failure, resulting in a stable quasi-static slipping patch propagation before reaching the nucleation time $t_{n}$ (or in normalized form $\sqrt{4 \alpha t_{n}} / a_{w}$ ), the shear crack tips are located well within the pressurized 


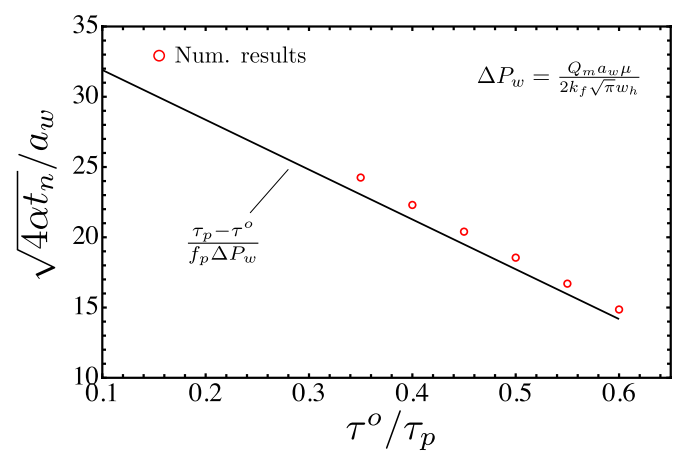

Figure 5: Comparison between the asymptotic solution (14) for ultimately stable faults valid for $t_{c} / t_{w} \ll 1$ and numerical solutions corresponding to the case of $t_{c} / t_{w}=0.01$ and $Q_{m} / Q^{*}=0.05$ (for which the necessary condition (15) is strictly satisfied for all values of $\tau^{o} / \tau_{p}$ considered).

region. At nucleation time, the rupture is not affected by the residual friction coefficient $f_{r}$ (due to the low slip accumulation during quasi-static slip propagation phase) and its half-length assumes the asymptotic expression (Garagash and Germanovich, 2012)

$$
\frac{a_{n}}{a_{w}} \simeq 0.579 \cdot \frac{\tau_{p}}{\tau^{o}}
$$

A careful investigation of Figure 4 that displays the corresponding slip, shear stress and friction coefficient profiles at different time snapshots (with dashed lines corresponding to the ones at nucleation time) and for two stress criticality values $\tau^{o} / \tau_{p}=0.4,0.6$ confirms these theoretical predictions. It is worth noting at the arrest of the dynamic slip propagation, the friction coefficient has reached its residual value over almost the entire crack. The subsequent shear crack propagation, therefore, remains always stable in time as depicted in Figure 3-left. In addition to this, Garagash and Germanovich (2012) proposed an asymptotic solution for the nucleation time $t_{n}$ that is valid for an ultimately stable fault that exhibits a dynamic event right after slip activation (similarly to the scenarios reported in Figure 3-left). Under this condition, indeed, the shear crack at nucleation time is confined near injection point and is subjected to a uniform over-pressure equal to the minimum value required to activate slip, i.e. $\bar{p}\left(x \simeq 0, t_{n}\right)=\left(\tau_{p}-\tau^{o}\right) / f_{p}$. This asymptotic 
solution reads (Garagash and Germanovich, 2012)

$$
\frac{\sqrt{4 \alpha t_{n}}}{a_{w}} \simeq \frac{\tau_{p}-\tau^{o}}{f_{p} \Delta P_{w}},
$$

where $\Delta P_{w}=\frac{Q_{m} a_{w} \mu}{2 k_{f} \sqrt{\pi} w_{h}}$ is the characteristic pore-pressure drop over the distance $a_{w}$. In order to guarantee that crack instability follows shortly after slip activation, $\Delta P_{w}$ must be very small compared to $\bar{p}\left(x \simeq 0, t_{n}\right)$, resulting in the following necessary condition

$$
\frac{Q_{m}}{Q^{*}} \ll \sqrt{\pi}\left(1-\frac{\tau^{o}}{\tau_{p}}\right)
$$

The comparison between the nucleation times associated with numerical simulations reported in Figure 3-left (for which $Q_{m} / Q^{*}=0.05$ strictly satisfies the condition (15) for each value of stress criticality considered) and the asymptotic solution (14) is displayed in Figure 5. A good agreement is obtained, specially for larger values of $\tau^{o} / \tau_{p}$ for which the assumption of nucleation after activation is truly valid (see Figure 3-left). Obviously, lower values of $Q_{m} / Q^{*}$ would lead to an earlier nucleation and thus a closer match between numerical and asymptotic solution.

For a larger value of injection rate $Q_{m} / Q^{*}=0.5$ (such to fall into region (1) of Figure 2 for $\tau^{o} / \tau_{p}<0.6$ ), instead, the slipping patch propagates always quasi-statically for all values of $\tau^{o} / \tau_{p}$, before reaching the maximum pressurization time $\sqrt{4 \alpha t_{\max }} / a_{w}$ at which the condition (12) is violated (and at which all the simulations were stopped). One exception is a very small seismic event corresponding to the boundary case of $\tau^{o} / \tau_{p}=0.6$ - see Figure 3-right.

These numerical results confirm that in case the maximum flow rate $Q_{m}$ is reached before pore fluid can actually diffuse into the fault, the hydromechanical fault response is essentially driven by pressurization at constant volumetric rate and a good match with theoretical results of Garagash and Germanovich (2012) is obtained. This is further strengthened by looking at the comparison between the numerical results reported in Figure 3 (black lines) and the small-scale yielding (s.s.y.) asymptotic solutions (dashed grey lines) associated with pressurization at constant injection rate only (see Appendix C). For $a / a_{w} \gtrsim 2$, indeed, a perfect match is obtained, suggesting that the effect of the quick ramp-up phase on slip propagation is negligible. 

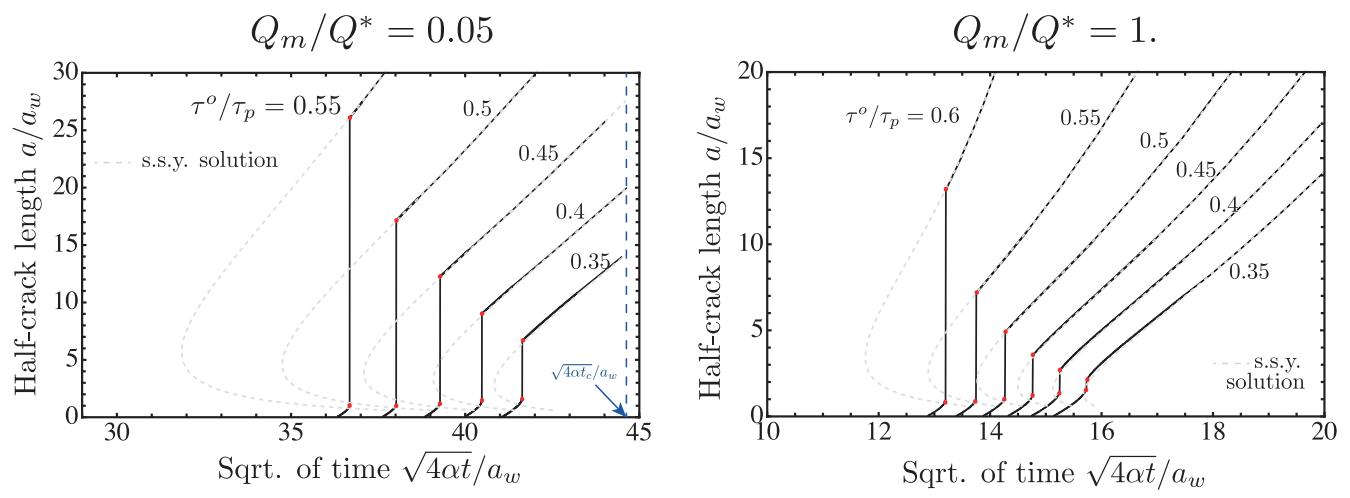

Figure 6: Time evolution of normalized half-crack length $a / a_{w}$ (black solid lines) as function of stress criticality $\tau^{o} / \tau_{p}$, for two values of maximum injection rates $Q_{m} / Q^{*}=0.05,1$ and $t_{c} / t_{w}=2 \cdot 10^{3} \gg 1$. The friction weakening ratio is set to $f_{r} / f_{p}=0.6$, resulting in ultimately stable conditions, i.e. $\tau^{o}<\tau_{r}$ for each value of ambient fault loading condition. Grey dashed lines represent the small scale yielding (s.s.y.) solution associated with linear ramp-up of injection rate (see Appendix $\mathrm{C}$ ), whose normalized ending time in this example is $\sqrt{4 \alpha t_{c}} / a_{w}=44.72$. Red dots, instead, denote the nucleation and arrest of dynamic slip.

\subsubsection{Slow ramp-up: the limit when $t_{c} / t_{w} \gg 1$}

We present here the opposite scenario in which injection rate increases linearly in time but the maximum value is reached after the pore fluid substantially diffuses along the fault plane (i.e. $t_{c} \gg t_{w}$ ). Under this condition, pore-pressure perturbation activates a shear crack (slip) during the ramp-up of injection rate, and the potential nucleation of a dynamic event would occur when pressurization is approaching the changing time $t_{c}$. In other words, $t_{c} \gg t_{w}$ and $t_{n} \lesssim t_{c}$.

Scaling analysis reported in Appendix B suggests that the pressurization rate, i.e. how quick is the ramp-up of injection rate before time $t_{c}$, does play a role in the hydro-mechanical fault response. In order to investigate its effect on the potential nucleation and arrest of dynamic slip on ultimately stable faults, we run several simulations keeping the ratio $t_{c} / t_{w}$ constant (and much larger than 1) and varying the maximum injection rate $Q_{m} / Q^{*}$. This is equivalent to keeping the normalized injection rate constant and varying the (large) ratio $t_{c} / t_{w}$, resulting in relatively low or large pressurization rates.

Figure 6 displays the time evolution of half-crack length for different values of stress criticality, a ratio $t_{c} / t_{w}=2 \cdot 10^{3}$ and two maximum injection rates $Q_{m} / Q^{*}=0.05,1$, representative of low and moderate fault pressurization 


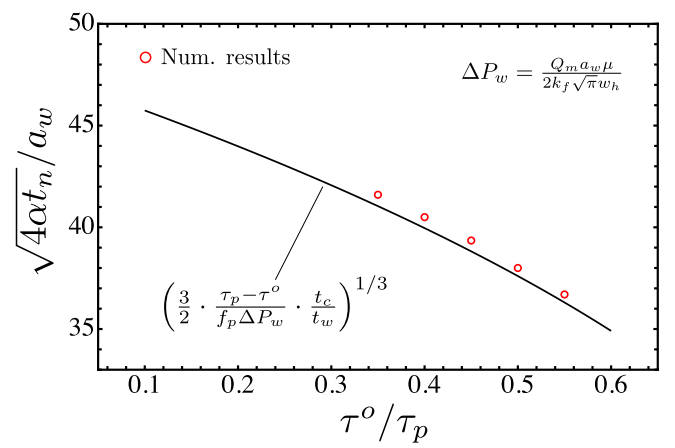

Figure 7: Comparison between the asymptotic analytical solution (16) for ultimately stable faults valid for $t_{c} / t_{w} \gg 1$ and numerical solutions corresponding to the case of $t_{c} / t_{w}=$ $2 \cdot 10^{3}$ and $Q_{m} / Q^{*}=0.05$ (for which the necessary condition (15) is strictly satisfied for all values of $\tau^{o} / \tau_{p}$ considered).

rates, respectively. Notice that the maximum injection rates considered are similar to the ones used in the previous case of $t_{c} / t_{w} \ll 1$ (see Figure 3 ), although the pressurization rates are considerably different. From a comparison of Figures 6-right and 3-right, we clearly observe that, in the case of $t_{c} / t_{w} \gg 1$, a moderate maximum injection rate is not sufficient to quench the finite-sized dynamic slip event for each value of stress criticality (unlike the case previously discussed). This is certainly due to the different type of fault pressurization that drives the slipping patch expansion. In this case, indeed, slip is driven by linear increase branch of injection rate and porepressure at injection point evolves proportional to $\sim t^{3 / 2}$ (see Eq. (10)). A further comparison of Figures 3-left and 6-left for the exact same value of $Q_{m} / Q^{*}=0.05$ reveals that the dynamic run-out distances are rather similar for each corresponding value of stress criticality, while the nucleation times differ considerably. With the similar approach of Garagash and Germanovich (2012), we derive an asymptotic expression for the nucleation time that is valid when nucleation of dynamic slip follows shortly shear crack activation. By setting $\bar{p}\left(\xi \simeq 0, t_{n}\right)$ from equation (10) equal to $\left(\tau_{p}-\tau^{o}\right) / f_{p}$, i.e. equal to the minimum over-pressure required to activate slip, we get the following asymptotic expression for the normalized nucleation time

$$
\frac{\sqrt{4 \alpha t_{n}}}{a_{w}} \simeq\left(\frac{3}{2} \cdot \frac{\tau_{p}-\tau^{o}}{f_{p} \Delta P_{w}} \cdot \frac{t_{c}}{t_{w}}\right)^{1 / 3} \Longleftrightarrow \frac{Q_{m}}{Q^{*}} \ll \sqrt{\pi}\left(1-\frac{\tau^{o}}{\tau_{p}}\right),
$$




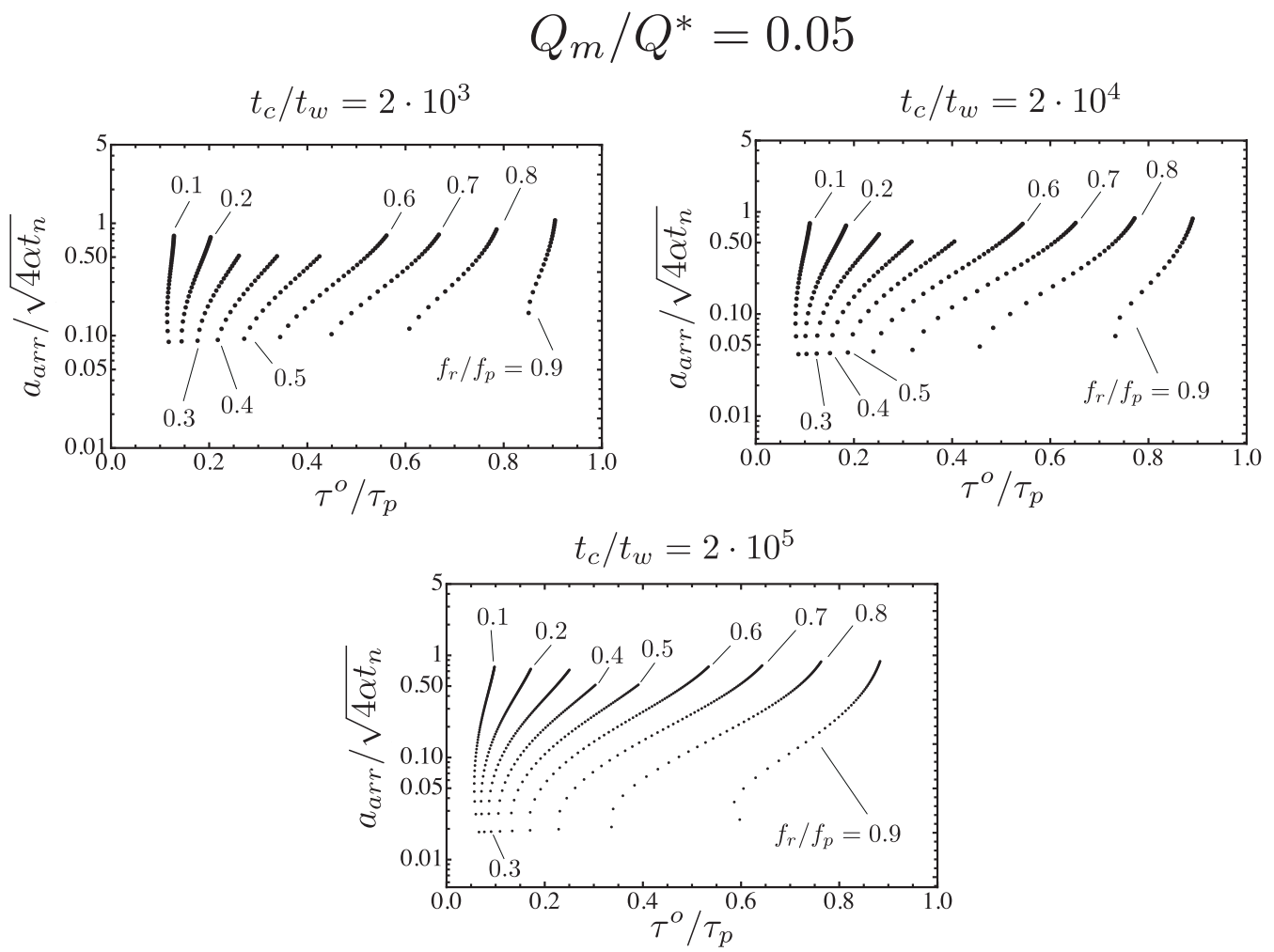

Figure 8: Normalized dynamic run-out distances $a_{a r r} / \sqrt{4 \alpha t_{n}}$ in function of stress criticality $\tau^{o} / \tau_{p}$ and friction weakening ratio $f_{r} / f_{p}$, for different ramp-up scenarios of injection rate, i.e. different values of $\frac{Q_{m} t_{w}}{t_{c} Q^{*}}$. These latter are obtained by fixing the maximum injection rate at $Q_{m} / Q^{*}=0.05$ and varying the (large) ratio $t_{c} / t_{w}$ (notably $\left.t_{c} / t_{w}=2 \cdot 10^{3}, 2 \cdot 10^{4}, 2 \cdot 10^{5} \gg 1\right)$. In all the cases nucleation occurs prior reaching time $t_{c}$, even for low values of $f_{r} / f_{p}$.

where $\Delta P_{w}$ is the same characteristic pore-pressure drop reported in Eq. (14). Unlike the asymptotic solution (14) valid for $t_{c} / t_{w} \ll 1$, in this case the normalized nucleation time varies non-linearly with stress criticality $\tau^{o} / \tau_{p}$, with a power law exponent equal to $1 / 3$. Furthermore, it appears the dependency on the dimensionless ratio $t_{c} / t_{w}$ with the same power law exponent (as expected from scaling analysis), revealing that the nucleation time of dynamic slip does depend on how quick the injection rate ramp-up occurs in time, and thus on how large is the ratio $\frac{Q_{m} \cdot t_{w}}{Q^{*} \cdot t_{c}}$. The comparison between the numerical results corresponding to the case of $Q_{m} / Q^{*}=0.05$, for which 
the condition $\frac{Q_{m}}{Q^{*}} \ll \sqrt{\pi}\left(1-\frac{\tau^{o}}{\tau_{p}}\right)$ is strictly satisfied for all the values of $\tau^{o} / \tau_{p}$, and the asymptotic solution (16) is displayed in Figure 7. A good agreement is obtained, with a closer match for larger values of $\tau^{o} / \tau_{p}$ due to the earlier nucleations after slip activations (see Figure 6-left).

The asymptotic solution (16) gives also an estimation of the normalized position of the fluid front at nucleation time. A careful inspection of Figure 6-left reveals that, at the onset of dynamic slip, the slipping patch front lags well within the pressurized region, and their relative distance decreases dramatically after the arrest of dynamic event (with fluid front always located ahead the slip front). Furthermore, we can observe that our numerical results (black lines) match perfectly with the small-scale yielding (s.s.y.) asymptotic solutions associated with linear increase of injection rate (dashed grey lines see Appendix C for more details) for each value of stress criticality. Since the extent of the arrested dynamic crack is always much larger than the slipping patch length scale $a_{w}$ (thus s.s.y. condition is truly valid) and the corresponding nucleation time is known analytically from (16), we can calculate analytically the dynamic run-out distances $a_{\text {arr }}$ directly from the small-scale yielding solution resolved at nucleation time. By simply replacing the nucleation time $t_{n}$ obtained from Eq. (16) into the increment of stress intensity factor associated with ramp-up of injection rate (C.3), we can solve the resulting implicit equation obtained from propagation criterion (C.6) for the unknown arrested crack lengths. Obviously, this analytical equation is only valid in the case of early dynamic crack nucleation after activation, but it allows to investigate systematically the effect of the other dimensionless parameters on the arrest of dynamic rupture.

We examine the effect of pressurization rate by fixing the maximum injection rate to $Q_{m} / Q^{*}=0.05$ (such to satisfy the necessary condition in Eq. (16) for the whole range of stress criticality $\tau^{o} / \tau_{p}$ ) and varying the large ratio $t_{c} / t_{w}$, obtaining thus a relatively quick or slow ramp-up of injection rate. In Figure 8, we display the normalized dynamic run-out distances $a_{a r r} / \sqrt{4 \alpha t_{n}}$ in function of stress criticality and friction weakening ratio $f_{r} / f_{p}$. We can observe that, for a given ramp-up of injection rate, the nucleation of a dynamic event is expected on a wider range of stress criticality for intermediate values of friction weakening ratio (compared to the cases of $f_{r} / f_{p} \rightarrow 0$ or $f_{r} / f_{p} \rightarrow 1$ ). Moreover, for a given value of $f_{r} / f_{p}$, the range of stress criticality in which a dynamic event is expected increases for increasing values of $t_{c} / t_{w}$ (i.e. for 


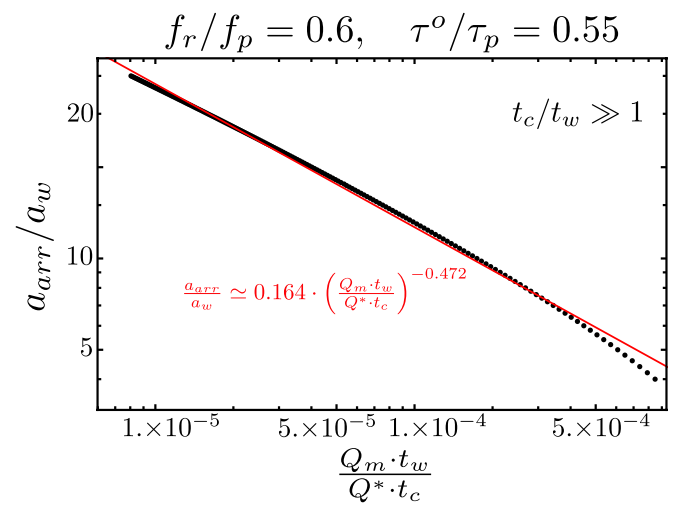

Figure 9: Normalized dynamic run-out distances $a_{a r r} / a_{w}$ (black dots) in function of pressurization rate $\frac{Q_{m} t_{w}}{t_{c} Q^{*}}\left(\right.$ with $t_{c} / t_{w} \gg 1$ ), for an ultimately stable fault characterised by $\tau^{o} / \tau_{p}=0.55$ and friction weakening ratio of $f_{r} / f_{p}=0.6$. The red solid line represents a linear fit of the numerical results (black dots) in the log-log plot.

decreasing values of $\frac{Q_{m} t_{w}}{t_{c} Q^{*}}$ ), revealing that low pressurization rates promote the nucleation of a finite-sized dynamic rupture on ultimately stable faults. It is also interesting to note that the arrest of the dynamic rupture always occurs within the pressurized region, i.e. $\frac{a_{\text {arr }}}{\sqrt{4 \alpha t_{n}}} \lesssim 1$, regardless of i) how quick the injection rate increase in time, ii) friction weakening ratio considered and iii) stress criticality value. Furthermore, for a given value of $f_{r} / f_{p}$ and $\tau^{o} / \tau_{p}$, the dynamic run-out distance increases for decreasing values of $\frac{Q_{m} t_{w}}{t_{c} Q^{*}}$, i.e. for slower ramp-up of injection rate. This can be grasped clearly from Figure 9 where the normalized ramp-up rate $\frac{Q_{m} t_{w}}{t_{c} Q^{*}}$ is plotted against the normalized dynamic run-out distance $a_{a r r} / a_{w}$ in a log-log plot. As one can see, the arrest of the dynamic slipping patch decreases non-linearly with increasing values of ramp-up rates, approximately proportional to an inverse power-law with exponent equal to -0.472 (see red line in Figure 9).

\subsection{Unstable fault $\left(\tau^{o}>\tau_{r}\right)$}

Finally, we present the case of injection into an unstable fault characterised by $\tau^{o} / \tau_{r}>1$ at ambient conditions. In this particular scenario the fault is critically stressed and thus prompt to fail. A small strength perturbation, due to for instance a small pore-pressure increment $\bar{p}$, always leads to 
a quick quasi-static shear crack propagation followed by a run-away dynamic rupture (albeit in some circumstances a finite-sized dynamic event may precede the run-away rupture - see region (3) of Figure 2 valid for the injection scenario at constant volumetric rate). Assuming a shear crack activation during the early ramp-up of injection rate (valid when $t_{c}$ is not much smaller than $t_{w}$ and thus pore fluid can diffuse within the fault plane), the slipping patch outpaces rapidly the pore fluid front and, at nucleation time $t_{n}$, the following condition hold

$$
\frac{a_{n}}{a_{w}} \gg \frac{\sqrt{4 \alpha t_{n}}}{a_{w}},
$$

with $t_{n}$ much smaller than both fluid diffusion time-scale $t_{w}$ and ramp-up time scale $t_{c}$. The pressurized region at nucleation time, therefore, is always confined near injection point and the corresponding pore-pressure distribution can be approximated using an equivalent point force distribution $\bar{p}(x, t) \simeq \Delta P(t) \delta_{\text {dirac }}(x)$, with $\Delta P(t)$ defined in (10).

Based on the previous work of Uenishi and Rice (2003), Garagash and Germanovich (2012) showed that there exist an asymptotic solution in terms of critical shear crack length that is universal (i.e. independent of the particular type of injection scenario) and it reads

$$
\frac{a_{n}}{a_{w}} \simeq 0.579
$$

In addition to this, they developed semi-analytically an outer and inner asymptotic solutions that are valid at different fault position with respect to fluid front location (see Appendix D). The outer solution is so called because is valid outside the pressurization region, i.e. for $|x| \gg \sqrt{4 \alpha t}$. The inner asymptotic solution, instead, is valid for $|x| \lesssim \sqrt{4 \alpha t}$, i.e. near injection point due to the limited extension of pressurization region on unstable faults before instability.

Since the outer asymptotic solution is universal, i.e. it is valid for any type of peak pore-pressure distribution (see Appendix D), we can make use of such a solution to derive an asymptotic expression for the nucleation time $t_{n}$ in function of the ramp-up of injection rate (and compare it with the solution of Garagash and Germanovich (2012) valid for pressurization at constant flow rate that can actually be retrieved here in the limit of $t_{c} \rightarrow 0$ ). Based on the outer solution, indeed, the integrated net over-pressure along the fault at crack instability is approximately given by (Garagash and Germanovich, 
2012)

$$
\Delta P\left(t_{n}\right)=\int_{-\infty}^{+\infty} \bar{p}\left(x, t_{n}\right) \mathrm{d} x \simeq \mathcal{P} \frac{\tau_{p}-\tau^{o}}{f_{p}} a_{w},
$$

where $\mathcal{P} \simeq 0.8369$ is the scaled magnitude of the point force, independent of the type of fault pressurization. By replacing Eq. (10) into Eq. (19), after some algebra, we obtain

$$
\frac{\sqrt{4 \alpha t_{n}}}{a_{w}} \simeq\left(\frac{2^{2} \cdot 0.8369}{\sqrt{\pi}} \cdot \frac{\tau_{p}-\tau^{o}}{f_{p} \Delta P_{w}} \cdot \frac{t_{c}}{t_{w}}\right)^{1 / 4},
$$

where $\Delta P_{w}$ is the same characteristic pore-pressure drop of Eq. (14). It is interesting to note that the normalized nucleation time in the case of an unstable fault subjected to a ramp-up of injection rate varies non-linearly with both stress criticality $\tau^{o} / \tau_{p}$ and pressurization rate $\frac{Q_{m} \cdot t_{w}}{Q^{*} \cdot t_{c}}$ (with a power law exponent equal to 1/4). A comparison with the normalized nucleation time associated with constant injection rate type of pressurization (Garagash and Germanovich, 2012)

$$
\frac{\sqrt{4 \alpha t_{n}}}{a_{w}} \simeq\left(\frac{2 \cdot 0.8369}{\sqrt{\pi}} \frac{\tau_{p}-\tau^{o}}{f_{p} \Delta P_{w}}\right)^{1 / 2}
$$

suggests that the ramp-up of injection rate prior the maximum plateau may promote an earlier or later nucleation of a run-away dynamic event, with respect to the injection scenario at constant volumetric rate. Indeed, by taking the ratio between equation (20) and equation (21), we obtain 1.20636 . $\left(\frac{f_{p} \Delta P_{w}}{\tau_{p}-\tau^{o}} \frac{t_{c}}{t_{w}}\right)^{1 / 4}$, implying that an earlier nucleation is expected when

$$
\frac{t_{c}}{t_{w}}<\frac{1}{1.20636^{4}} \frac{\tau_{p}-\tau^{o}}{f_{p} \Delta P_{w}}
$$

for a given maximum value of injection rate $Q_{m}$.

In order to verify all these theoretical predictions, we run several numerical simulations with different stress criticality values, such to obtain highly critically stressed fault conditions for which condition (17) is strictly satisfied. We fixed the maximum injection rate at $Q_{m} / Q^{*}=100$, the friction weakening ratio at $f_{r} / f_{p}=0.6$ and the normalized changing time at $t_{c} / t_{w}=0.5$. As we can observe from Figure 10, the quick quasi-static shear crack propagation 

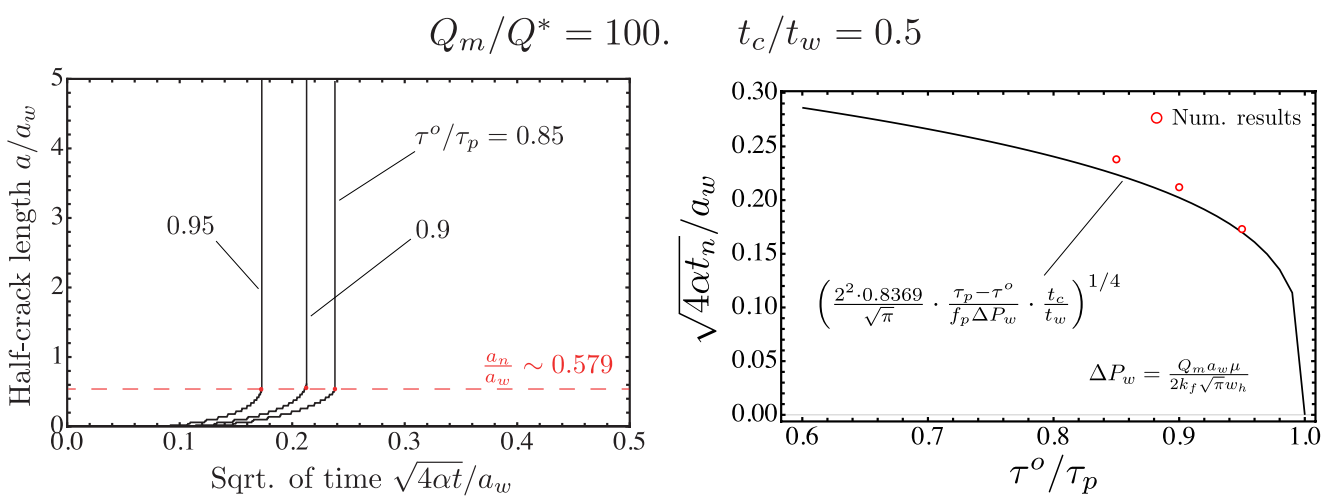

Figure 10: Left: time evolution of normalized half-crack length $a / a_{w}$ for different values of (large) stress criticality $\tau^{o} / \tau_{p}=0.95,0.9,0.85$. Since the friction weakening ratio is $f_{r} / f_{p}=0.6$, all the scenarios correspond to very unstable faults. The maximum injection rate is set to $Q_{m} / Q^{*}=100$, while the normalized ramp-up time is $t_{c} / t_{w}=0.5$ (or in terms of normalized square root of time $\sqrt{4 \alpha t_{c}} / a_{w} \simeq 0.71$ ). Right: comparison between the asymptotic analytical solution (20) and the numerical results in terms of normalized nucleation time $\sqrt{4 \alpha t_{n}} / a_{w}$, for each value of $\tau^{o} / \tau_{p}$ considered (see left plot).

is always followed by a run-away dynamic rupture, whose nucleation time is in good agreement with theoretical prediction of Eq. (20) (with a better accuracy for larger values of $\tau^{o} / \tau_{p}$ due to a more strict validity of condition (17)). At the instability, the normalized crack length $a / a_{w}$ is approximately $\sim 0.579$ for each value of stress criticality considered (as expected) and the corresponding scaled slip distributions match very well the outer and inner asymptotic solutions at $|x| \gg \sqrt{4 \alpha t}$ and $|x| \lesssim \sqrt{4 \alpha t}$, respectively (see Figures D.12 and D.13 in Appendix D).

\section{Discussions}

In this section, we first discuss the limitations of our modelling approach and their effects on nucleation and arrest of dynamic fault slip. After that, we discuss the implications of our results on injection-induced seismicity, a topic that has raised the attention of scientific community and public opinion since second half of $20^{\text {th }}$ century (Simpson, 1986).

\subsection{Model limitations}

The most severe approximation in our model is the neglect of dilatancy during fluid-driven shear crack propagation. The assumption of impermeable 
host medium around fault gouge unit accommodating slip and pore-fluid diffusion is representative of an immature deep fault, typically characterised by rough inner surfaces. Strong dilatant behavoir associated with sliding over fault's asperities has been observed in laboratory experiments (Lockner and Byerlee, 1994; Samuelson et al., 2009) as well as during field experiments in the context of geothermal energy exploitation (Batchelor, 1985). Under the assumption of no fluid leak-off in the relative undamaged rock around the fault, shear-induced dilatancy does affect pore-pressure evolution, which in turn leads to a feedback on slip propagation (due to full coupling between flow and elastic deformations). Under undrained conditions, indeed, shearinduced dilatancy leads to a pore-pressure drop and thus to a local increase of effective normal stress (dilatant hardening) (Rudnicki, 1979; Segall and Rice, 1995). Ciardo and Lecampion (2019) have shown that such a dilatant hardening effect, quantified by a scaled undrained pore-pressure drop $\frac{\Delta w_{h}}{w_{h} c_{f} \sigma_{o}^{\prime}}$ with $\Delta w_{h}$ being the increment of fault opening, does impact the transition between aseismic and seismic slip on frictional weakening immature faults. They showed, in fact, that a large dilatant behaviour suppresses the nucleation of run-away seismic rupture on otherwise unstable faults, even for sustained increases of fault permeability with slip. On ultimately stable faults, instead, dilatant hardening effect delays the nucleation of finite-sized seismic event and increases its dynamic run-out distance. We can certainly say, however, that the findings obtained in this work are valid for an immature fault whose dilatant compliance is relatively small, i.e. for $\frac{\Delta w_{h}}{w_{h} c_{f} \sigma_{o}^{\prime}} \ll 0.1$ which would result in an undrained pore-pressure drop of fractions of MPa.

In this contribution we also neglect other weakening mechanisms that can kick in during the onset of dynamic crack propagation, such as thermal pressurization and flash heating (Rice, 2006).

Thermal pressurization of pore-fluid by rapid shear heating of fault gouge unit has been showed to be a prominent process of fault weakening (Viesca and Garagash, 2015). This mechanism depends on fluid thermodynamics properties and drives the shear strength loss at low fluid pressure conditions (Acosta et al., 2018). Garagash and Germanovich (2012) showed that dynamic weakening due to thermal pressurization increases the dynamic run-out slip distances on ultimately stable faults. However, they also stated that such an effect is relatively small, due to the fact the most of dynamic weakening is expected to occur at slip scale $\delta_{w, \text { dyna }}$ that is likely larger than $\delta_{w}$. Without 
loss of generality, we can claim that the results presented in this contribution in terms of dynamic run-out distances on ultimately stable faults are valid for sufficiently low values $\delta_{w}$ compared to the characteristic dynamic slipweakening distance $\delta_{w, \text { dyna }}$ (which depends on heat properties of both fault gouge unit and injected fluid).

Flash heating on fault's asperities, instead, kicks in when the slip velocity exceeds $\sim 0.1 \mathrm{~m} / \mathrm{s}$, a scenario that is very plausible during dynamic crack propagation, which characteristic slip rate at the rupture tip typically exceeds $\sim 10 \mathrm{~m} / \mathrm{s}$ (Garagash, 2011). Laboratory experiments on various types of rocks have shown a drop of friction coefficient of up to one order of magnitude due to flash heating on asperities contacts (Di Toro et al., 2011). These considerations suggest that this weakening mechanism would impact the dynamic run-out distances presented in this contribution. However, this is outside the scope of this work and it is left for future investigations.

Finally, we adopted a simple linear slip-weakening friction law compared to a more elaborate Rate- and State- friction model (Dieterich, 1979). Although a number of studies have demonstrated that the linear slip-weakening friction model is a good approximation of the velocity weakening R\&S friction law (Uenishi and Rice, 2003; Rubin and Ampuero, 2005; Garagash, 2021) even at slip instability (Viesca, 2016b,a), it does not allow to investigate scenarios in which fault frictional properties evolve during pressurization, for instance from velocity weakening to velocity strengthening depending on current slip velocity (as observed in laboratory experiments by Cappa et al. (2019)). Indeed, this can only be captured by using R\&S friction law with heterogeneous distribution of $a$ and $b$ parameters. Future works with incorporation of R\&S friction model will follow.

\subsection{Implications on injection-induced seismicity}

The results above may indicate that injection-induced seismicity can be mitigated by controlling operational parameters, in particular the injection volumetric rate.

Upon fluid injection into a specific fracture zone, possibly indicating of a well-developed fault zone, pore-pressure perturbation is likely to activate slip on favourably oriented (and critically stressed) fractures within the damage zone. The initial stable slip propagation could be quickly followed by runaway seismic ruptures that in turn could trigger events on other fractures and propagate over the entire fault zone. Our results show that the onset of the contained micro-seismicity, however, may be delayed in time for slow 
increases of injection rate prior reaching a steady-state phase. If a specific stable principal fault plane is targeted for fluid injection, instead, a larger pore-fluid perturbation is required to activate slip. A ramp-up of the injection rate, in this case, strongly affects the slip propagation, in particular when the ramp-up time scale is much larger than the along-fault diffusion time scale. Although counter-intuitive, a fast ramp-up of injection rate on stable fault planes would reduce the possibility of triggering a larger finitesized seismic event (with respect to the one that could be triggered if fault pressurization occurs at constant injection rate), which can potentially turn into an unabated rupture due to the activation of other dynamic weakening mechanisms (as discussed in Section 4.1).

The results are essentially in line with previous laboratory and numerical observations. Indeed, previous results highlighted how the initial state of effective stress (Gischig, 2015; Passelègue et al., 2018) and essentially its relationship to the frictional behaviour (Larochelle et al., 2021) control whether a fault slip is confined to the pressurized region or runaways. Similarly, the numerical analysis by Alghannam and Juanes (2020) reports how the pressurization rate may influence the seismic reactivation of a fault zone. Here we generalize both approaches by showing that the ruptures and its final behaviour (confined or runaway) are linked to both the initial state of stress as well as the initial ramp-up of the injection, which is then closely linked to the pressurization rate. The effects of the controlling operational parameters on risk of induced-seismicity associated with the specific physic-based model presented in this contribution are summarized in Figure 11. It displays the effects of the initial ramp-up of injection rate on the different slip regimes (the same of Figure 2), as function of all the other governing dimensionless parameters. A comparison with Fig. 2 that is valid for the simple injection scenario of constant rate reveals that, for an ultimately stable fault in which $t_{c} / t_{w} \gg 1$, the larger is the pressurization rate (i.e. the larger is the ratio $\frac{Q_{m} t_{w}}{t_{c} Q^{*}}$ ), the smaller is region (2) and thus the lower is the possibility of triggering a finite-sized seismic event. For slightly unstable faults $\left(\tau^{o} \gtrsim \tau_{r}\right)$, instead, a lower pressurization rate would lead to a larger slip accumulation during the quick quasi-static phase of crack propagation. The friction coefficient, therefore, would drop quicker to its residual value and the probability of triggering a run-away dynamic rupture preceded by a finite-sized event is higher, resulting in a larger region (3). This also implies that the nucleation time of such a run-away rupture increases for lower pressurization rates, as 


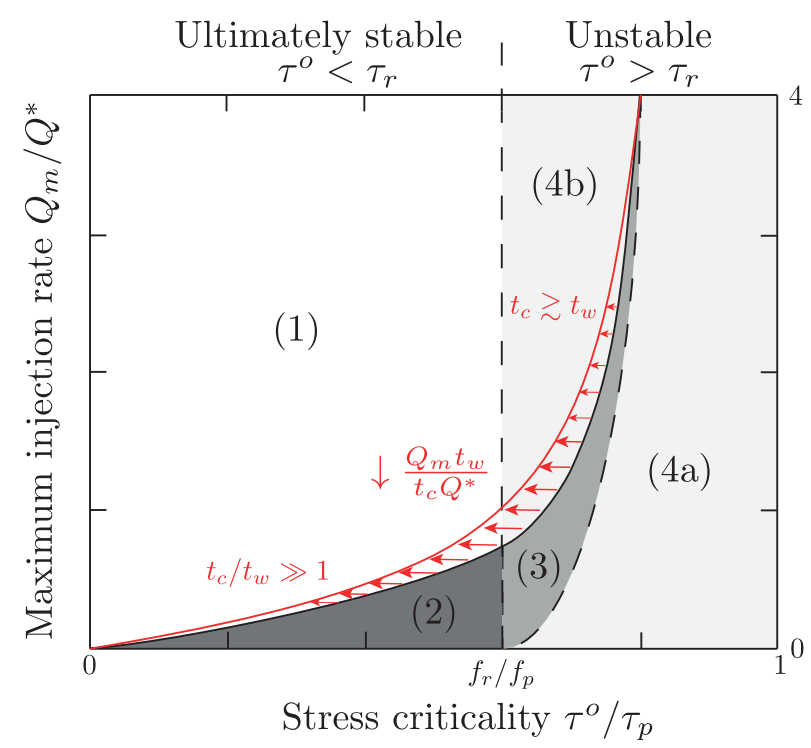

Figure 11: Map of slip regimes for a frictional weakening pressurized fault in which injection rate increases linearly in time, up to reach a maximum plateau after time $t_{c}$. This parametric diagram is function of stress criticality $\tau^{o} / \tau_{p}$, friction weakening ratio $f_{r} / f_{p}$, normalized maximum injection rate $Q_{m} / Q^{*}$ and normalized ramp-up time scale $t_{c} / t_{w}$. For a given value of $Q_{m} / Q^{*}$, the larger is the ratio $t_{c} / t_{w}$, i.e. the lower is the pressurization rate $\frac{Q_{m} t_{w}}{t_{c} Q^{*}}$, the more wide are region (2) and (3). All the slip regimes of this Figure are the same of those reported and described in Figure 2.

more and more part of the shear crack has reached the residual shear strength during its quasi-static stable propagation.

\section{Conclusions}

In this study, we have extended the model of Garagash and Germanovich (2012) to account for an initial ramp-up of injection rate in time before the maximum plateau and investigated its effect on nucleation and arrest of dynamic fault slip. Despite the simplicity of the homogeneous model (planar bidimensional fault, uniform stress conditions and rock properties, linear weakening of friction, no dilatancy), it allows to get insight into the mechanisms that govern the transition between aseismic and seismic slip on a pressurized planar fault.

We have approximated standard injection protocols that consist of an initial step-wise ramp-up of injection rate in time with a linear increasing function, 
followed by a maximum plateau after a given time $t_{c}$. We have solved numerically the coupled hydro-mechanical problem and explored the different slip regimes identified via scaling analysis. Our results show that the initial ramp-up of injection rate affects the slip propagation on ultimately stable faults $\left(\tau^{o}<\tau_{r}\right)$ if and only if the ramp-up time scale $t_{c}$ is much larger than fluid diffusion time scale $t_{w}$. Notably, we have shown that slip stability is governed by the pressurization rate (quantified by the dimensionless parameter $\left.\frac{Q_{m} t_{w}}{Q^{*} t_{c}}\right)$ and thus by how quickly the injection rate increases before reaching time $t_{c}$. From our results we can conclude that low pressurization rates applied on ultimately stable faults with frictional weakening properties and $t_{c} \gg t_{w}$ promote the nucleation of a finite-sized dynamic event. Moreover, the lower is the pressurization rate, the larger is the dynamic runt-out slip distance and hence the larger is the magnitude of the induced seismic event. We have also developed an asymptotic solution in terms of nucleation time that is valid when slip instability follows shortly crack activation and verified it with numerical simulations.

When $t_{c} \ll t_{w}$, instead, the initial ramp-up of injection rate can be neglected on ultimately stable faults and slip are driven by pressurization at constant volumetric rate. A good agreement with theoretical predictions of Garagash and Germanovich (2012) valid for that particular injection condition has been obtained.

Finally, we have demonstrated that the ramp-up of injection rate does affect the quick slip propagation on critically stressed faults prior the nucleation of a run-away dynamic rupture. By using the universal outer asymptotic solution of Garagash and Germanovich (2012), we developed a new analytical solution for the nucleation time and verified it with numerical simulations. This solution reveals that the initial ramp-up of injection rate may lead to an earlier or later nucleation of unabated dynamic event, compared to the case of fault pressurization at constant volumetric rate.

\section{Appendix A. Along-fault pore-pressure diffusion: analytical solu- tion}

Equation (5) or equivalently equation (9) is a parabolic, linear secondorder partial differential equation with constant coefficients. It governs the one-dimensional over-pressure diffusion $\bar{p}(x, t)=p(x, t)-p_{o}$ inside the fault gouge unit with constant hydraulic diffusivity $\alpha\left[L^{2} / T\right]$. Together with the 
boundary conditions

$$
\bar{p}( \pm \infty, t)=0, \quad-\left.\frac{w_{h} k_{f}}{\mu} \frac{\partial \bar{p}}{\partial x}\right|_{x=0^{ \pm}}= \pm\left\{\begin{array}{ll}
\frac{Q_{m}}{2 t_{c}} \cdot t & t \leq t_{c} \\
\frac{Q_{m}}{2} & t>t_{c}
\end{array},\right.
$$

and the specific initial conditions, it represents a well-posed diffusion problem that can be solved analytically (Carslaw and Jaeger, 1959).

Firstly, we solve the diffusion problem for the case of linear ramp-up of injection rate valid for $t \leq t_{c}$, for which the initial condition reads

$$
\bar{p}(x, 0)=0
$$

We use Laplace transform in time (with Laplace parameter $s$ ) in order to turn equation (9) into an ordinary differential equation (with transformed variables denoted with an hat $\left.{ }^{\wedge}\right)$. With the initial condition (A.2), such a subsidiary equation reads

$$
\frac{\partial^{2} \hat{p}(x, s)}{\partial x^{2}}-k^{2} \hat{p}(x, s)=0 \quad \text { with } \quad k^{2}=\frac{s}{\alpha},
$$

whose analytical solution is

$$
\hat{p}(x, s)=c_{1}(s) \cdot \mathrm{e}^{k x}+c_{2}(s) \cdot \mathrm{e}^{-k x}
$$

$c_{1}(s)$ and $c_{2}(s)$ are two constants that can be obtained in closed form using the boundary conditions (A.1) associated only with linear ramp-up of injection rate:

$$
c_{1}(s)=0, \quad c_{2}(s)=-\frac{\mu Q_{m}}{2 t_{c} k_{f} s^{2} w_{h} k}
$$

By taking the inverse Laplace transform of equation (A.4) with the constants defined in equation (A.5), we get the analytical expression for alongfault over-pressure diffusion reported in Eq. (10) and valid for $t \leq t_{c}$, i.e.

$$
\bar{p}\left(\xi, t \leq t_{c}\right)=\left(\frac{2 Q_{m} \mu \sqrt{\alpha} t^{3 / 2}}{3 k_{f} \sqrt{\pi} t_{c} w_{h}}\right) \cdot\left(\mathrm{e}^{-\xi^{2}}\left(1+\xi^{2}\right)-\sqrt{\pi}|\xi|\left(\frac{3}{2}+\xi^{2}\right) \operatorname{Erfc}(|\xi|)\right)
$$

798 where $\xi=\frac{x}{\sqrt{4 \alpha t}}$ is the along-fault normalized coordinate and Erfc is the complementary error function. 
We then solve the pore-fluid diffusion problem for time $t>t_{c}$, taking into account the injection history during the ramp-up phase.

In view of problem linearity, the analytical expression for the over-pressure evolution valid for time $t>t_{c}$ can be obtained by solving the diffusion equation (9) using as initial condition the over-pressure distribution evaluated at time $t_{c}$ (using Eq. (A.6)), and as boundary conditions the relations (A.1) associated only with constant injection rate. By using the fundamental heat conduction solution valid for an infinite one-dimensional body subjected to an instantaneous point source (also called Green's function) (Carslaw and Jaeger, 1959)

$$
G\left(x-x^{\prime}, t-t^{\prime}\right)=\frac{1}{\sqrt{4 \pi \alpha\left(t-t^{\prime}\right)}} \mathrm{e}^{-\frac{\left(x-x^{\prime}\right)^{2}}{4 \alpha\left(t-t^{\prime}\right)}} \quad \text { for } \quad t-t^{\prime} \geq 0
$$

and the superimposition principle, we can express the over-pressure evolution valid for time $t>t_{c}$ via the following analytical formula (Cole et al., 2011):

$\bar{p}\left(x, t>t_{c}\right)=\int_{-\infty}^{\infty} G\left(x-x^{\prime}, t-t_{c}\right) \cdot \bar{p}\left(\frac{x^{\prime}}{\sqrt{4 \alpha t_{c}}}, t_{c}\right) \mathrm{d} x^{\prime}+\frac{\alpha Q_{m} \mu}{w_{k} k_{f}} \int_{t^{\prime}=t_{c}}^{t} G\left(x, t-t^{\prime}\right) \mathrm{d} t^{\prime}$

Notice that the first term of Eq. (A.8) is the convolution with respect to the variable $x$ of the fundamental solution (A.7) and the over-pressure distribution at time $t_{c}$ (obtained from (A.6)), while the second term is the contribution due to the constant flux boundary condition at fault centre. If the ramp-up time $t_{c}$ vanishes (i.e. $t_{c}=0$ ), then fault pressurization would occur at constant injection rate. The first member of equation (A.8) would vanish (due to the initial condition (A.2)) and the analytical solution for the over-pressure evolution along the fault plane upon integration would read as

$$
\bar{p}(x, t)=\frac{\alpha Q_{m} \mu}{w_{k} k_{f}} \cdot \frac{t \cdot E_{\frac{3}{2}}\left(\frac{x^{2}}{4 t \alpha}\right)}{2 \sqrt{\pi} \sqrt{\alpha t}},
$$

where $E_{n}(z)$ is the Exponential integral function, or expressed in a similar form of (A.6) as

$$
\bar{p}(\xi, t)=\left(\frac{Q_{m} \mu \sqrt{\alpha t}}{\sqrt{\pi} k_{f} w_{h}}\right) \cdot\left(\mathrm{e}^{-\xi^{2}}-\sqrt{\pi}|\xi| \operatorname{Erfc}(|\xi|)\right)
$$

with $\xi=\frac{x}{\sqrt{4 \alpha t}}$. 


\section{Appendix B. Scaling analysis}

Scaling analysis applied on physics-based models represents a powerful technique that can help a systematic investigation of all the physical processes occurring. For sake of completeness, we report here the dimensionless solution structure as well as the normalised set of governing equations.

Based on previous work of Garagash and Germanovich (2012), we introduce the following characteristic scales in order to normalize elasticity equation (1) and shear stress evolution within the crack tips (3):

$\tau(x, t)=\tau_{p} \cdot \mathcal{T}(x, t), \quad \delta(x, t)=\delta_{w} \cdot \Delta(x, t), \quad \bar{p}(x, t)=\sigma_{o}^{\prime} \cdot \Pi(x, t) \quad x=a \cdot \mathcal{X}$

where $\delta_{w}$ is the slip weakening length-scale (see Figure 1), $\tau_{p}=f_{p} \sigma_{o}^{\prime}$ is the peak shear strength at ambient conditions and $a$ is half-length of the shear crack. The corresponding governing equations upon introduction of (B.1) read:

$$
\mathcal{T}(a \mathcal{X}, t)=\frac{\tau^{o}}{\tau_{p}}-\frac{1}{2 \pi} \frac{a_{w}}{a} \int_{-1}^{1} \frac{\partial \Delta(a \mathcal{X}, t)}{\partial \zeta} \frac{\mathrm{d} \zeta}{(\zeta / a-\mathcal{X})}
$$

$$
\mathcal{T}(a \mathcal{X}, t)=\frac{f(\delta)}{f_{p}}(1-\Pi(a \mathcal{X}, t))
$$

where $a_{w}=\frac{E_{p}}{2 \tau_{p}} \delta_{w}$ is a characteristic length-scale of the slipping patch and the normalized friction coefficient evolution is defined as

$$
\frac{f(\delta)}{f_{p}}= \begin{cases}1-\Delta(a \mathcal{X}, t) & \Delta(a \mathcal{X}, t) \leq 1-\frac{f_{r}}{f_{p}} \\ \frac{f_{r}}{f_{p}} & \Delta(a \mathcal{X}, t)>1-\frac{f_{r}}{f_{p}}\end{cases}
$$

In order to normalize the fluid diffusion problem (5-8), we use the same characteristic scales introduced in (B.1), with the exception that now we scale the spatial coordinate $x$ with the length-scale $a_{w}$ previously obtained. Time $t$, instead, is normalized with the diffusion time-scale $t_{w}=\frac{a_{w}^{2}}{4 \alpha}$ such that

$$
t=t_{w} \cdot \mathrm{T}
$$

Equations (5-8), therefore, reduce to

$$
\frac{\partial \Pi}{\partial \mathrm{T}}-\frac{1}{4} \frac{\partial^{2} \Pi}{\partial \mathcal{X}^{2}}=0
$$


844

$$
\left.\frac{\partial \Pi}{\partial \mathcal{X}}\right|_{\mathcal{X}=0^{ \pm}}= \begin{cases} \pm \frac{Q_{m}}{Q^{*}} \frac{t_{w}}{t_{c}} \cdot \mathrm{T} & \mathrm{T} \leq \frac{t_{c}}{t_{w}} \\ \pm \frac{Q_{m}}{Q^{*}} & \mathrm{~T}>\frac{t_{c}}{t_{w}}\end{cases}
$$

where $Q^{*}$ is the characteristic scale of maximum injection rate and is defined as

$$
Q^{*}=\frac{2 \sigma_{o}^{\prime} w_{h} k_{f}}{a_{w} \mu}
$$

Inspecting equations (B.2-B.4) and (B.6-B.7) we can readily observe that the dimensionless solution is function of only four dimensionless parameters:

- $\frac{\tau^{o}}{\tau_{p}} \rightarrow$ fault stress criticality

- $\frac{f_{r}}{f_{p}} \rightarrow$ friction weakening ratio

- $\frac{Q_{m}}{Q^{*}} \rightarrow$ normalized maximum injection rate

- $\frac{t_{c}}{t_{w}} \rightarrow$ normalized ramp-up time scale

\section{Appendix C. Small-Scale Yielding asymptotics}

When half-length of slipping patch $a$ is sufficiently larger than the characteristic length-scale $a_{w}$, the model non-linearity (i.e. slip weakening of friction coefficient) is localized only over a region that is small compared to geometrical dimensions of the rupture zone. A clear example of such a condition, typically named as small-scale yielding, is depicted in Figure 4bottom where, after the arrest of dynamic slip, the friction coefficient has not reached its residual value $f_{r}$ only over a small region near crack tips. The elastic stress-intensity factor, therefore, controls the local deformation field when $a \gg a_{w}$ (Rice, 1968), and its analytical expression can be obtained in closed form by superimposing the effects of net loading applied on the shear crack (i.e. far-field stress $\tau^{o}$ minus residual shear tractions $\tau_{r}$ ) and of fluid pressurization as (Tada et al., 2000)

$$
K_{I I}=\left(\tau^{o}-\tau_{r}\right) \sqrt{\pi a}+\Delta K_{I I},
$$


where

$$
\Delta K_{I I}=f_{r} \sqrt{\frac{a}{\pi}} \int_{-a}^{+a} \frac{\bar{p}(x, t)}{\sqrt{a^{2}-x^{2}}} \mathrm{~d} x
$$

By replacing the analytical expression for over-pressure evolution during the ramp-up phase (10) into (C.2), we find that the increment of stressintensity factor due to pressurization at time $t \leq t_{c}$ is

$$
\begin{aligned}
\Delta K_{I I}=-\frac{f_{r} \sqrt{a} \mu Q_{m} t e^{-\frac{a^{2}}{8 \alpha t}}}{36 \sqrt{\pi} k_{f} t_{c} w_{h}(\alpha t)^{3 / 2}} & \left(4 a^{3} \sqrt{\alpha t} e^{\frac{a^{2}}{8 \alpha t}}+36 a(\alpha t)^{3 / 2} e^{\frac{a^{2}}{8 \alpha t}}-\sqrt{\pi} a^{4} I_{0}\left(\frac{a^{2}}{8 t \alpha}\right)-\right. \\
& -9 \sqrt{\pi} a^{2} \alpha t I_{0}\left(\frac{a^{2}}{8 t \alpha}\right)-\sqrt{\pi} a^{4} I_{1}\left(\frac{a^{2}}{8 t \alpha}\right)-11 \sqrt{\pi} a^{2} \alpha t I_{1}\left(\frac{a^{2}}{8 t \alpha}\right)- \\
& -3 \sqrt{\pi} a^{2} \alpha t e^{\frac{a^{2}}{8 \alpha t}} \cosh \left(\frac{a^{2}}{8|\alpha||t|}\right) I_{0}\left(\frac{a^{2}}{8|t||\alpha|}\right)- \\
& -24 \sqrt{\pi} \alpha^{2} t^{2} e^{\frac{a^{2}}{8 \alpha t}} \cosh \left(\frac{a^{2}}{8|\alpha||t|}\right) I_{0}\left(\frac{a^{2}}{8|t||\alpha|}\right)+ \\
& +3 \sqrt{\pi} a^{2}|\alpha||t| e^{\frac{a^{2}}{8 \alpha t}} \cosh \left(\frac{a^{2}}{8|\alpha||t|}\right) I_{1}\left(\frac{a^{2}}{8|t||\alpha|}\right)+ \\
& +3 \sqrt{\pi} a^{2}|\alpha \| t| e^{\frac{a^{2}}{8 \alpha t}} \sinh \left(\frac{a^{2}}{8|\alpha||t|}\right) I_{0}\left(\frac{a^{2}}{8|t||\alpha|}\right)+ \\
& +24 \sqrt{\pi} \alpha t|\alpha||t| e^{\frac{a^{2}}{8 \alpha t}} \sinh \left(\frac{a^{2}}{8|\alpha||t|}\right) I_{0}\left(\frac{a^{2}}{8|t||\alpha|}\right)- \\
& \left.-3 \sqrt{\pi} a^{2} \alpha t e^{\frac{a^{2}}{8 \alpha t}} \sinh \left(\frac{a^{2}}{8|\alpha||t|}\right) I_{1}\left(\frac{a^{2}}{8|t||\alpha|}\right)\right)
\end{aligned}
$$

where $I_{n}(z)$ is the modified Bessel function of the first kind.

If pressurization consists of injection at constant flow rate only, then the increment of stress-intensity factor would be obtained by replacing equation (A.10) into (C.2), leading to the following expression

$\Delta K_{I I}=-\frac{e^{-\frac{a^{2}}{8 \alpha t}} \sqrt{a} f_{r} \mu Q_{m}}{4 \sqrt{\pi} k_{f} w_{h} \sqrt{\alpha t}}\left(4 a e^{\frac{a^{2}}{8 \alpha t}} \sqrt{\alpha t}-\sqrt{\pi} a^{2} I_{0}\left(\frac{a^{2}}{8 t \alpha}\right)-\sqrt{\pi} a^{2} I_{1}\left(\frac{a^{2}}{8 t \alpha}\right)-4 \sqrt{\pi} \alpha t I_{0}\left(\frac{a^{2}}{8 t \alpha}\right)\right)$

During the slipping patch propagation driven by pore-fluid diffusion, the energy release rate $G=\frac{K_{I I}^{2}}{E_{p}}$ must be equal to the fracture energy $G_{c}$, whose 
analytical expression under the assumption of small-scale yielding and constant effective normal stress near crack tips is (Palmer and Rice, 1973)

$$
G_{c} \simeq\left(f_{p}-f_{r}\right) \sigma^{\prime}(a) \frac{\delta_{r}}{2}
$$

The quasi-static propagation criterion

$$
G=G_{c}
$$

therefore provides an asymptotic solution for the slipping patch length $a$ as function of pressurization time $t$.

Following the previous work of Dempsey et al. (2010), however, Garagash and Germanovich (2012) have shown that by replacing the slipping patch length $a$ in (C.1-C.2) by an effective (reduced) length $a_{\text {eff }}$, a more accurate expression of the stress-intensity factor in the limit of $a \gg a_{w}$ is obtained. Such an effective crack length $a_{e f f}$ is function of a process zone size $d$ that Dempsey et al. (2010) determined numerically for the case of cohesive crack with linear softening traction separation law and propagating under uniform far-field tractions (which share the same mathematical formulation), i.e.

$$
a_{e f f} \simeq a-0.466 \cdot d,
$$

where $d$ is defined as

$$
d \simeq 0.466 \cdot \lambda
$$

and $\lambda=\left(\frac{\pi}{2}\right) \cdot\left(\frac{K_{I I}}{\left(\tau_{p}-\tau_{r}\right)}\right)^{2}$ is a characteristic length-scale.

In this contribution, the implicit equation (C.6) incorporates the reduced crack length (C.7) and it is solved numerically for time $t$ (thus imposing the variable crack length $a$ ) by minimizing the residual function using a random search method. When the slipping patch is driven essentially by pressurization at constant injection rate (for instance in the case of an ultimately stable fault in the limit when $t_{c} / t_{w} \ll 1$ ), equation (C.6) is solved using the stressintensity factor obtained in (C.4). Instead, when the shear crack is driven up to the nucleation of a dynamic event by the ramp-up of injection rate (for instance in the case of an ultimately stable fault with $t_{c} / t_{w} \gg 1$ ), then equation (C.6) is solved using (C.3) (obviously up to the maximum value $t_{c}$ ). The comparison between the numerical results (black lines) and small-scale asymptotic solutions (dashed grey lines) in Figures 3 and 6 shows a good match for slipping patches $a$ larger than $\sim 2 a_{w}$. 


\section{Appendix D. Outer and inner asymptotic solutions at instability for an unstable fault}

Here we briefly report the outer and inner asymptotic solutions at instability for an unstable fault $\tau^{o} / \tau_{r}>0$ developed by Garagash and Germanovich (2012). The former is universal, which means it is independent of a particular pore-pressure profile, while the latter does depend on the particular pore-pressure distribution. Since dynamic instability for an unstable fault occurs quickly after fluid injection and crack activation (and thus during the ramp-up of injection rate for values of $t_{c}$ comparable to or greater than $t_{w}$ ), the inner asymptotic solution is solved only for the particular distribution (10).

\section{Appendix D.1. Outer solution}

At instability, the slipping patch extent is much larger than the pressurized region, due to the rapid propagation of the shear crack after its activation. Under this condition, the pore pressure distribution can be replaced by an equivalent point-force distribution

$$
\bar{p}(x, t) \simeq \Delta P(t) \delta_{\text {dirac }}(x)
$$

where $\delta_{\text {dirac }}$ is the delta Dirac function and $\Delta P(t)=\int_{-\infty}^{\infty} \bar{p}(x, t) \mathrm{d} x$. This equivalent distribution, however, approximates well $\bar{p}(x, t)$ only for distances much larger than fluid front position, i.e. for $x \gg \sqrt{4 \alpha t}$ (outside pressurization region).

Garagash and Germanovich (2012) solved semi-analytically the hydro-mechanical problem with the equivalent pore-pressure distribution (D.1) and proved that the normalized slip profile at instability is given by

$$
\frac{\delta(x)}{\epsilon \delta_{w}}=\mathcal{P}\left(\bar{\delta}_{1}(X)-\frac{a}{a_{w}} \bar{\delta}_{2}(X)\right)+\bar{\delta}(X),
$$

where $\mathcal{P} \simeq 0.8369$ is the scaled magnitude of the point force, $\epsilon=1-\tau^{o} / \tau_{p}$ is the understress parameter, $X=x / a$ is the normalized fault coordinate, and 


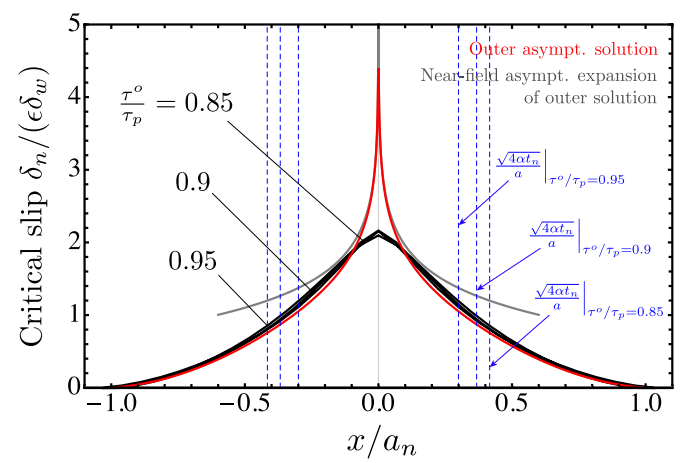

Figure D.12: Corresponding normalized slip distributions $\delta_{n} /\left(\epsilon \delta_{w}\right)$ (black solid lines) at nucleation time $t_{n}$ of the numerical simulations reported in Figure 10. The red solid line denotes the outer asymptotic solution valid outside the pressurization regions (whose extents at instability are identified by blue dashed lines), i.e. for $\left|\frac{x}{a_{n}}\right| \gg \frac{\sqrt{4 \alpha t_{n}}}{a_{n}}$, while the grey solid line represents the near-field expansion of the outer asymptotic solution that is valid for $x / a_{n} \ll 1$.

$\bar{\delta}_{1}(X), \bar{\delta}_{2}(X)$, and $\bar{\delta}(X)$ are three functions that are defined as

$$
\begin{aligned}
& \bar{\delta}_{1}(X)=\frac{2}{\pi} \cdot \ln \left(\frac{1+\sqrt{1-X^{2}}}{|X|}\right) \\
& \bar{\delta}(X) \simeq-1.1732 \cdot \sin (\operatorname{arcos}(X))-0.0608 \cdot \sin (3 \cdot \operatorname{arcos}(X))+0.0235 \cdot \sin (5 \cdot \operatorname{arcos}(X)) \\
& \bar{\delta}_{2}(X)=\frac{2}{\pi} \int_{-1}^{1} \ln \left|\frac{X-s}{1-s X+\sqrt{1-s^{2}} \sqrt{1-X^{2}}}\right| \bar{\delta}_{1}(s) \mathrm{d} s
\end{aligned}
$$

In Eq. (D.3), $\bar{\delta}(X)$ is a continuous and differentiable function whose analytical expression has been obtained numerically using Gauss-Chebyshev polynomial quadrature (with truncation at third term - see (Garagash and Germanovich, 2012) for more details), while $\bar{\delta}_{2}(X)$ is the inverse of the Cauchy integral in terms of $\bar{\delta}(X)$ (solved numerically in this contribution). Equation (D.2) represents a good approximation of slip distribution only for $\left|\frac{x}{a}\right| \gg \frac{\sqrt{4 \alpha t}}{a}$. A near-field asymptotic expansion of (D.2), however, is proved to be a good approximation of the critical distribution of normalized slip $\delta /\left(\epsilon \delta_{w}\right)$ for $X \ll 1$ and $a=a_{n}$ (Garagash and Germanovich, 2012). Such 


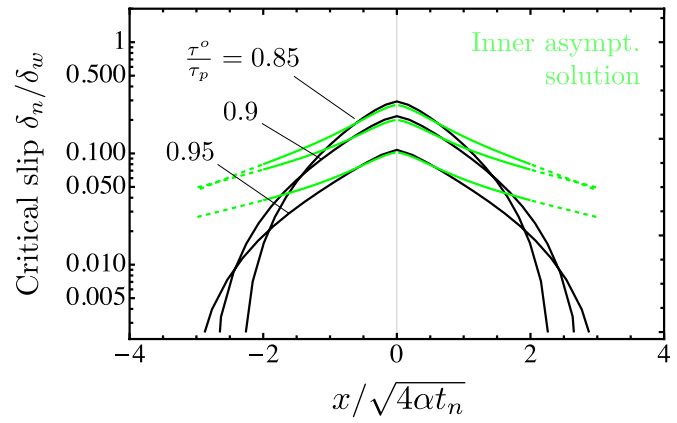

Figure D.13: Linear-log plot showing the corresponding normalized slip distributions $\delta_{n} / \delta_{w}$ (black solid lines) at nucleation time $t_{n}$ of the numerical simulations reported in Figure 10. The green solid lines denote the inner asymptotic solutions valid within the pressurization regions, i.e. for $\left|\frac{x}{\sqrt{4 \alpha t_{n}}}\right| \lesssim 1$.

an expansion is given as

$$
\frac{\delta(x)}{\epsilon \delta_{w}}=-\frac{2}{\pi} \mathcal{P} \ln \frac{|X|}{2}-\frac{a}{a_{w}} \mathcal{P} \bar{\delta}_{2}(0)+\bar{\delta}(0)+\mathcal{O}(X),
$$

where the ratio $a_{n} / a_{w}$ is defined in (18).

In order to check the accuracy of these theoretical predictions, we display in Figure D.12 the corresponding slip profiles at nucleation time $t_{n}$ of the numerical simulations reported in Fig. 10. As we can observe, the scaled slip distributions $\delta_{n} /\left(\epsilon \delta_{w}\right)$, which collapse into nearly one black line due to the scaling adopted, match the outer asymptotic solution (D.2) (denoted by a red solid line) for $\left|x / a_{n}\right| \gg \sqrt{4 \alpha t_{n}} / a_{n}$, i.e. outside the pressurization regions whose extents are identified by blue dashed lines (for a given value of stress criticality $\left.\tau^{o} / \tau_{p}\right)$. For $x / a_{n} \ll 1$, instead, the scaled slip distributions tend to converge to the near-field asymptotic expansion of the outer solution (D.4) (as expected).

\section{Appendix D.2. Inner solution}

Within the small pressurization region, i.e. for $x \lesssim \sqrt{4 \alpha t}$, the normalized pore-pressure distribution is approximately $\bar{p}(x) / \sigma_{o}^{\prime} \sim 1$, which means that the stress perturbation can be written as (Garagash and Germanovich, 2012)

$$
\frac{\tau(x)-\tau^{o}}{\tau_{p}} \simeq-\frac{\bar{p}(x)}{\sigma_{o}^{\prime}}+\mathcal{O}(\epsilon)
$$


for $\epsilon \ll 1$. Using this condition, Garagash and Germanovich (2012) showed that the normalized slip distribution at instability $\delta_{n} / \delta_{w}$ for a particular pore-pressure profile $\Psi(s)$ is given as

$$
\frac{\delta_{n}(\xi)}{\delta_{w}}=\frac{\delta_{n}(0)}{\delta_{w}}-\frac{2}{\pi} \epsilon \mathcal{P} \frac{\int_{-\infty}^{\infty} \Psi(s) \ln |1-\xi / s| \mathrm{d} s}{\int_{-\infty}^{\infty} \Psi(s) \mathrm{d} s}
$$

where $\delta_{n}(0)$ is the critical slip at $\xi=\frac{x}{4 \alpha t}=0$, which can be obtained by matching the outer $(x \gg \sqrt{4 \alpha t})$ and inner $(x \lesssim \sqrt{4 \alpha t})$ asymptotic solutions at intermediate distances (Garagash and Germanovich, 2012)

$$
\frac{\delta_{n}(0)}{\delta_{w}}=0.533 \epsilon\left(-\ln \frac{\hat{\epsilon}}{C}+1.003\right)
$$

where $\hat{\epsilon}=\left(\tau_{p}-\tau^{o}\right) / f_{p} \Delta \bar{p}\left(t_{n}\right)$ and the constant $C$ is defined as

$$
C=\left(\int_{-\infty}^{\infty} \Psi(s) \mathrm{d} s\right) \cdot \operatorname{Exp}\left(-\frac{\int_{-\infty}^{\infty} \Psi(s) \ln |s| \mathrm{d} s}{\int_{-\infty}^{\infty} \Psi(s) \mathrm{d} s}\right)
$$

Unlike the outer asymptotic solution, the inner solution does depend on the particular pore-pressure profile $\Psi(s)$. By replacing the instantaneous spatial distribution (10) associated with ramp-up of injection rate, we obtain $C=3.75576$, allowing us to calculate the normalized slip distribution using (D.7) and (D.6) (with numerical evaluation of the integral $\left.\int_{-\infty}^{\infty} \Psi(s) \ln |1-\xi / s| \mathrm{d} s\right)$. For the same injection condition, friction weakening ratio and initial loading conditions of the simulations reported in Figure 10, we show in Figure D.13 the corresponding normalized slip distributions $\delta_{n} / \delta_{w}$ at nucleation time $t_{n}$ on a linear-log plot. We can observe that, for each value of (large) stress criticality $\tau^{o} / \tau_{p}$, the corresponding inner asymptotic solution matches well the numerical results (back solid lines) for $|x| / \sqrt{4 \alpha t_{n}} \lesssim 1$ (as expected).

\section{Acknowledgments}

This project has been subsidised through the ERANET Cofund GEOTHERMICA (Project No. 200320-4001) by the Swiss Federal Office of Energy (SFOE), which is supported by the European Union's HORIZON 2020 programme for research, technological development and demonstration. Technical review comments by Elias Heimisson at SED are greatly appreciated. 
The analytical formulae and numerical methods described in the main text and appendices are sufficient to reproduce all the results presented in the paper.

\section{Authors' contributions}

F.C. contributed to the Conceptualization, Methodology, Software, Validation, Formal analysis, Investigation, Visualization, Writing original draft. A.P.R. contributed to Editing, Supervision.

\section{Conflict of interest}

The authors declare that they have no conflict of interest.

\section{References}

Acosta, M., Passelègue, F.X., Schubnel, A., Violay, M., 2018. Dynamic weakening during earthquakes controlled by fluid thermodynamics. Nature communications 9 .

Alghannam, M., Juanes, R., 2020. Understanding rate effects in injectioninduced earthquakes. Nature communications 11.

Almakari, M., Dublanchet, P., Hervé, E., Pellet, F., 2019. Effect of the injection scenario on the rate and magnitude content of injection-induced seismicity: Case of a heterogeneous fault. Journal of Geophysical Research: Solid Earth 124, 8426-8448.

Amann, F., Gischig, V., Evans, K., Doetsch, J., Jalali, R., Valley, B., Krietsch, H., Dutler, N., Villiger, L., Brixel, B., Klepikova, M., Kittilä, A., Madonna, C., Wiemer, S., Saar, M., Loew, S., Driesner, T., Maurer, H., Giardini, D., 2018. The seismo-hydromechanical behavior during deep geothermal reservoir stimulations: Open questions tackled in a decameter-scale in situ stimulation experiment. Solid Earth 9, 115-137. doi:10.5194/se-9-115-2018.

Barbour, A., Norbeck, J.H., Rubinstein, J.L., 2017. The effects of varying injection rates in Osage County, Oklahoma, on the 2016 Mw 5.8 Pawnee earthquake. Seismological Research Letters 88, 1040-1053. 
Batchelor, A.S., 1985. Hot dry rock reservoir stimulation in the UK an extended summary, in: International seminar on the results of EC geothermal energy research. 3, European Commission, München. pp. 681-711.

Brace, W., Kohlstedt, D., 1980. Limits on lithospheric stress imposed by laboratory experiments. Journal of Geophysical Research: Solid Earth 85, $6248-6252$.

Cappa, F., Scuderi, M.M., Collettini, C., Guglielmi, Y., Avouac, J.P., 2019. Stabilization of fault slip by fluid injection in the laboratory and in situ. Science advances 5 .

Carslaw, H.S., Jaeger, J.C., 1959. Conduction of heat in solids. Oxford Univ Press.

Chang, K.W., Yoon, H., Martinez, M.J., 2018. Seismicity rate surge on faults after shut-in: Poroelastic response to fluid injection. Bulletin of the Seismological Society of America 108, 1889-1904.

Chen, K.H., Bürgmann, R., 2017. Creeping faults: Good news, bad news? Rev. Geoph. 55, 282-286.

Ciardo, F., Lecampion, B., 2019. Effect of dilatancy on the transition from aseismic to seismic slip due to fluid injection in a fault. Journal of Geophysical Research: Solid Earth 124, 3724-3743.

Ciardo, F., Lecampion, B., Fayard, F., Chaillat, S., 2020. A fast boundary element based solver for localized inelastic deformations. Int. J. Numer. Meth. Engng. , 1-23.

Cole, K.D., Haji-Sheikh, A., Beck, J.V., Litkouhi, B., 2011. Heat conduction using Green's functions. Taylor \& Francis.

Deichmann, N., Giardini, D., 2009. Earthquakes Induced by the Stimulation of an Enhanced Geothermal System below Basel (Switzerland). Seismological Research Letters 80, 784-798.

Dempsey, D., Riffault, J., 2019. Response of Induced Seismicity to Injection Rate Reduction: Models of Delay, Decay, Quiescence, Recovery, and Oklahoma. Water Resources Res. 55, 656-681. 
Dempsey, J.P., Tan, L., Wang, S., 2010. An isolated cohesive crack in tension. Continuum Mechanics and Thermodynamics 22, 617-634.

Di Toro, G., Han, R., Hirose, T., De Paola, N., Nielsen, S., Mizoguchi, K., Ferri, F., Cocco, M., Shimamoto, T., 2011. Fault lubrication during earthquakes. Nature 471.

Diehl, T., Clinton, J., Kraft, T., Husen, S., Plenkers, K., Guilhelm, A., Behr, Y., Cauzzi, C., Kästli, P., Haslinger, F., Fäh, D., Michel, C., Wiemer, S., 2014. Earthquakes in Switzerland and surrounding regions during 2013. Swiss Geological Society 107, 359-357.

Diehl, T., Kraft, T., Kissling, E., Wiemer, S., 2017. The induced earthquake sequence related to the St. Gallen deep geothermal project (Switzerland): Fault reactivation and fluid interactions imaged by microseismicity. Journal of Geophysical Research: Solid Earth 122, 7272-7290.

Dieterich, J.H., 1979. Modeling of rock friction: 1. experimental results and constitutive equations. Journal of Geophysical Research: Solid Earth 84, 2161-2168.

Edwards, B., Kraft, T., Cauzzi, C., Kästli, P., Wiemer, S., 2015. Seismic monitoring and analysis of deep geothermal projects in St Gallen and Basel, Switzerland. Geophys. J. Int. 201, 1022-1039.

Ellsworth, W.L., 2013. Injection-induced earthquakes. Science 341.

French, M., Zhu, W., Banker, J., 2016. Fault slip controlled by stress path and fluid pressurization rate. Geophys. Res. Letters 43, 4330-4339.

Garagash, D., 2011. On fracture energy of flash heating, in: AGU Fall Meeting Abstracts, pp. T13A-2360.

Garagash, D.I., 2021. Fracture mechanics of rate-and-state faults and fluid injection induced slip. Phil. Trans. R. Soc. A 379. Https://doi.org/10.1098/rsta.2020.0129.

Garagash, D.I., Germanovich, L.N., 2012. Nucleation and arrest of dynamic slip on a pressurized fault. Journal of Geophysical Research 117. 
Garagash, D.I., Rudnicki, J.W., 2003. Shear heating of a fluid-saturated slip-weakening dilatant fault zone, 1, limiting regimes. J. Geophys. Res. 108.

Giardini, D., 2009. Geothermal quake risks must be faced. Nature 462, 848-849.

Gischig, V., 2015. Rupture propagation behavior and the largest possible earthquake induced by fluid injection into deep reservoirs. Geophys. Res. Lett. 42, 7420-7428.

Goertz-Allmann, B.P., Goertz, A., Wiemer, S., 2011. Stress drop variations of induced earthquakes at the Basel geothermal site. Geophysical Research Letters 38.

Grawinkel, A., Stockhert, B., 1997. Hydrostatic pore fluid pressure to $9 \mathrm{~km}$ depth - Fluid inclusion evidencefrom the KTB deep drill hole. Geophys. Res. Letters 24, 3273-3276.

Grigoli, F., Cesca, S., Rinaldi, A.P., Manconi, A., López-Comino, J.A., Clinton, J.F., Westaway, R., Cauzzi, C., Dahm, T., Wiemer, S., 2018. The November 2017 Mw 5.5 Pohang earthquake: A possible case of induced seismicity in South Korea. Science 360, 1003-1006.

Häring, M.O., Schanz, U., Ladner, F., Dyer, B.C., 2008. Characterisation of the Basel 1 enhanced geothermal system. Geothermics 37, 469-495.

Hofmann, H., Zimmerman, G., Zang, A., Min, K.B., 2018. Cyclic soft stimulation (CSS): a new fluid injection protocol and traffic light system to mitigate seismic risks of hydraulic stimulation treatments. Geothermal Energy 6.

Holland, A.A., 2013. Earthquakes triggered by hydraulic fracturing in southcentral Oklahoma. Bulletin of the Seismological Society of America 103, 1784-1792.

Horton, S., 2012. Disposal of hydrofracking waste fluid by injection into subsurface aquifers triggers earthquake swarm in central Arkansas with potential for damaging earthquake. Seismological Research Letters 83, 250-260. 
Ito, T., Zoback, M.D., 2000. Fracture permeability and in situ stress to $7 \mathrm{~km}$ depth in the KTB Scientific Drillhole. Geophys. Res. Letters 27, 1045-1048.

Keranen, K.M., Savage, H., Abers, G., Cochran, E.S., 2013. Potentially induced earthquakes in Oklahoma, USA: Links between wastewater injection and the $2011 \mathrm{Mw} 5.7$ earthquake sequence. Geology 41, 699-702.

Keranen, K.M., Weingarten, M., Abers, G., Bekins, B., Ge, S., 2014. Sharp increase in central Oklahoma seismicity since 2008 induced by massive wastewater injection. Science 345, 448-451.

Kim, K.H., Ree, J.H., Kim, Y., Kim, S., Kang, S.Y., Seo, W., 2018. Assessing whether the $2017 \mathrm{Mw} 5.4$ Pohang earthquake in South Korea was an induced event. Science, eaat6081.

Kim, W.Y., 2013. Induced seismicity associated with fluid injection into a deep well in Youngstown, Ohio. Journal of Geophysical Research: Solid Earth 118, 3506-3518.

Lagenbruch, C., Zoback, M.D., 2016. How will induced seismicity in Oklahoma respond to decreased saltwater injection rates? Science advances 2 .

Larochelle, S., Lapusta, N., Ampuero, J.P., Cappa, F., 2021. Constraining fault friction and stability with fluid-injection field experiments. Geophys. Res. Lett. 48.

Levandowski, W., Weingarten, M., Ill, R.W., 2018. Geomechanical sensitivities of injection-induced earthquakes. Geophys. Res. Letters 45, 8958-8965.

Lockner, D.A., Byerlee, J.D., 1994. Dilatancy in hydraulically isolated faults and the suppression of instability. Geophysical Research Letters 21, 23532356 .

Noël, C., Passelègue, F.X., Giorgetti, C., Violay, M., 2019. Fault reactivation during fluid pressure oscillations: transition from stable to unstable slip. Journal of Geophysical Research: Solid Earth 124.

Palmer, A.C., Rice, J.R., 1973. The growth of slip surfaces in the progressive failure of over-consolidated clay, in: Proceedings of the Royal Society of 
London A: Mathematical, Physical and Engineering Sciences, The Royal Society. pp. 527-548.

Passelègue, F.X., Almakari, M., Dublanchet, P., Barras, F., Fortin, J., Violay, M., 2020. Initial effective stress controls the nature of earthquakes. Nature communications 11 .

Passelègue, F.X., Brantut, N., Mitchell, T.M., 2018. Fault reactivation by fluid injection: Controls from stress state and injection rate. Geophys. Res. Lett. 54, 12837-12846.

Qin, Y., Chen, X., Walter, J.I., Haffener, J., Trugman, D.T., Carpenter, B.M., Weingarten, M., Kolawole, F., 2019. Deciphering the stress state of seismogenic faults in Oklahoma and Southern Kansas based on an improved stress map. Journal of Geophysical Research: Solid Earth 124.

Quarteroni, A., Sacco, R., Saleri, F., 2000. Numerical Mathematics. Texts in applied mathematics, Springer.

Rice, J.R., 1968. Mathematical analysis in the mechanics of fracture, in: H. Liebowitz (Ed.), Fracture: An Advanced Treatise. volume 2. chapter 3, pp. 191-311.

Rice, J.R., 1980. The mechanics of earthquake rupture. Physics of the Earth's Interior , 555-649.

Rice, J.R., 1992. Fault Stress States, Pore Pressure Distributions, and the Weakness of the San Andreas Fault. International Geophysics 51, 475-503.

Rice, J.R., 2006. Heating and weakening on faults during earthquake slip. J. Geophys. Res 111.

Rubin, A., Ampuero, J.P., 2005. Earthquake nucleation on (aging) rate and state faults. Journal of Geophysical Research: Solid Earth 110.

Rudnicki, J.W., 1979. The stabilization of slip on a narrow weakening fault zone by coupled deformation-pore fluid diffusion. Bull. Seism. Soc. Am. 69, 1011-1026.

Rudnicki, J.W., Zhan, Y., 2020. Effect of pressure rate on rate and state frictional slip. Geophys. Res. Letters 47. 
Samuelson, J., Elsworth, D., Marone, C., 2009. Shear-induced dilatancy of fluid-saturated faults: Experiment and theory. Journal of Geophysical Research: Solid Earth 114.

Segall, P., Rice, J.R., 1995. Dilatancy, compaction, and slip instability of a fluid-infiltrated fault. Journal of Geophysical Research: Solid Earth 100, 22155-22171.

Segall, P., Rubin, A., Rice, J.R., Schmitt, S., 2008. Dilatancy stabilization vs thermal pressurization as a mechanism for controlling slow vs fast slip. AGU Fall Meeting Abstracts .

Simpson, D.W., 1986. Triggered earthquakes. Annu. Rev. Earth Planet. Sci. $14,21-42$.

Sumy, D.F., Cochran, E.S., Keranen, K.M., Wei, M., Abers, G., 2014. Observations of static Coulomb stress triggering of the November 2011 M5.7 Oklahoma earthquake sequence. Journal of Geophysical Research: Solid Earth 119, 1904-1923.

Suppe, J., 2014. Fluid overpressures and strength of the sedimentary upper crust. Journal of Structural Geology 69, 481-492.

Tada, H., C., P.P., Irwin, G.R., 2000. The Stress Analysis of Cracks Handbook. 3rd ed.

Uenishi, K., Rice, J.R., 2003. Universal nucleation length for slip-weakening rupture instability under nonuniform fault loading. Journal of Geophysical Research: Solid Earth 108.

Viesca, R.C., 2016a. Self-similar slip instability on interfaces with rate-and state-dependent friction. Proc. Roy. Soc. Lond. A 472, 20160254.

Viesca, R.C., 2016b. Stable and unstable development of an interfacial sliding instability. Physical Review E 93, 060202.

Viesca, R.C., Garagash, D.I., 2015. Ubiquitous weakening of faults due to thermal pressurization. Nature Geoscience 8, 875 .

Villiger, L., Gischig, V.S., Doetsch, J., Krietsch, H., Dutler, N.O., Jalali, M., Valley, B., Selvadurai, P.A., Mignan, A., Plenkers, K., Giardini, D., 
Amann, F., Wiemer, S., 2020. Influence of reservoir geology on seismic response during decameter-scale hydraulic stimulations in crystalline rock. Solid Earth 11, 627-655.

Wang, L., Kwiatek, G., Rybacki, E., Bonnelye, A., Bohnhoff, M., Dresen, G., 2020. Laboratory study on fluid-induced fault slip behavior: The role of fluid pressurization rate. Geophysical Research Letters 47 .

Warpinski, N.R., Teufel, L.W., 1987. Influence of geologic discontinuities on hydraulic fracture propagation. Journal of Petroleum Technology 39, 209-220.

Weingarten, M., Ge, S., Godt, J.W., Bekins, B.A., Rubinstein, J.L., 2015. High-rate injection is associated with the increase in U.S. mid-continent seismicity. Science 348, 1336-1340.

Wibberley, C.A.J., 2002. Hydraulic diffusivity of fault gouge zones and implications for thermal pressurization during seismic slip. Earth Planets Space 54, 1153-1171.

Wibberley, C.A.J., Yielding, G., Di Toro, G., 2008. Recent advances in the understanding of fault zone internal structure: A review. Geological Society Special Publication 299, 5-33.

Wong, T.F., 1986. On the normal stress dependence of the shear fracture energy. Earthquake Source Mechanics, Geophysical Monograph Series 37.

Yeo, I.W., Brown, M.R.M., Ge, S., Lee, K.K., 2020. Causal mechanism of injection-induced earthquakes through the $\mathrm{Mw} 5.5$ Pohang earthquake case study. Nature communications 11.

Zhang, X., Jeffrey, R.G., Llanos, E.M., 2005. On plane-strain fluid-driven shear fracture propagation in elastic solids. Geophys. J. Int. 163, 419-430.

Zoback, M.D., Zoback, M.L., Mount, V.S., Suppe, J., Eaton, J.P., Healy, J.H., Oppenheimer, D., Reasenberg, P., Jones, L., Raleigh, C.B., Wong, I.G., Scotti, O., Wentworth, C., 1987. New evidence on the state of stress of the San Andreas fault system. Science 238, 1105-1111. 